\title{
Adhesion dynamics of confined membranes
}

\author{
Tung B.T. To, ${ }^{a, b}$ Thomas Le Goff, ${ }^{a, c}$ and Olivier Pierre-Louis ${ }^{a, \dagger}$
}

\begin{abstract}
We report on the modeling of the dynamics of confined lipid membranes. We derive a thin film model in the lubrication limit which describes an inextensible liquid membrane with bending rigidity confined between two adhesive walls. The resulting equations share similarities with the Swift-Hohenberg model. However, inextensibility is enforced by a time-dependent nonlocal tension. Depending on the excess membrane area available in the system, three different dynamical regimes, denoted as A, B and C, are found from the numerical solution of the model. In regime A, membranes with small excess area form flat adhesion domains and freeze. Such freezing is interpreted by means of an effective model for curvature-driven domain wall motion. The nonlocal membrane tension tends to a negative value corresponding to the linear stability threshold of flat domain walls in the Swift-Hohenberg equation. In regime B, membranes with intermediate excess areas exhibit endless coarsening with coexistence of flat adhesion domains and wrinkle domains. The tension tends to the nonlinear stability threshold of flat domain walls in the Swift-Hohenberg equation. The fraction of the system covered by the wrinkle phase increases linearly with the excess area in regime $\mathrm{B}$. In regime $\mathrm{C}$, membranes with large excess area are completely covered by a frozen labyrinthine pattern of wrinkles. As the excess area is increased, the tension increases and the wavelength of the wrinkles decreases. For large membrane area, there is a crossover to a regime where the extrema of the wrinkles are in contact with the walls. In all regimes after an initial transient, robust localised structures form, leading to an exact conservation of the number of adhesion domains.
\end{abstract}

\section{Introduction}

Bilayer lipid membranes are abundant in biological systems ${ }^{1}$. They are found in cell membranes, skin, eyes, articulations and pulmonary organs 2 . Since their elasticity is dominated by bending rigidity, lipid membranes exhibit specific morphologies and dynamics, classifying their shape in a different class as compared to surface tension dominated phenomena, which govern the physics of capillarity and wetting. Helfrich has first proposed a model energy functional within which the behavior of lipid membranes could be explored 5 . In the past two decades, much work has been devoted to the analysis of the consequences of the Helfrich energy on the morphology and dynamics of lipid membranes. Various successes include the shape of vesicles at equilibrium ${ }^{6}$ or under hydrodynamic flow 78 , or the behavior of membrane stacks, and of supported membranes on substrates 9.11 .

Here we wish to investigate the consequences of confinement on the dynamics of membranes. We study the dynamics of a fluid membrane with bending rigidity and area conservation confined between two attractive walls. This geometry is firstly motivated by the suggestion that membranes can experience a double-well adhesion potential in cell adhesion processes $\frac{12}{2}$, or in biomimetic experiments 13 . In these experiments, a short-range potential well located in the vicinity of the substrate results from the molecular binding of ligand-receptor pairs. In addition, a free energy barrier at intermediate ranges is provided by the entropic repulsion of a polymer brush grafted to the substrate, which mimics the glycocalyx 14 . Finally, a long range attraction, resulting either from Van der Waals forces $\frac{12}{12}$, or from gravity 13 enforces a second po-

\footnotetext{
${ }^{a}$ Institut Lumière Matière, UMR5306 Université Lyon 1-CNRS, Université de Lyon, 69622 Villeurbanne, France

${ }^{b}$ Instituto de Física, Universidade Federal Fluminense, Avenida Litorânea s/n, 24210340 Niterói, RJ, Brazil

${ }^{c}$ Aix Marseille Univ, CNRS, IBDM-UMR7288, 13009 Marseille, France

† Olivier.Pierre-Louis@univ-lyon1.fr
}

tential well for the membrane. In parallel with these works, other studies in the literature allow one to formulate a different picture for membrane adhesion, which is also based on the concept of a double-well potential. Indeed, blebbing 15 - the local detachment of cell membranes from the cytoskeleton, suggests that the link between membranes and the cytoskeleton can be described to some extent by simple adhesion concepts. Cell adhesion could therefore be mimicked by a competition between this adhesion of the membrane to the cytoskeleton, and adhesion to a substrate. Such a scenario is reminiscent of that proposed in Ref. 18 , where membranes experience competing adhesion between a substrate and the cytoskeleton. Moreover, a third and somewhat different situation involving a double-well potential arises when two types ligand-receptor pairs with different lengths compete for adhesion, leading to two possible equilibrium separation distances, as proposed in Ref. 19 .

Our study is inspired by these diverse pictures of membrane adhesion in two-state, or double-well potentials. Our aim here is to capture some generic features of these systems by exploring the simple case of a membrane confined between two flat adhesive walls. Adhesion in biological cells involving e.g. signaling, the remodeling of the cytoskeleton, or the diffusion and clustering of ligands and receptors, is certainly more complex than our minimal modeling approach. However, we hope that our results will provide hints to understand systems involving more physical ingredients.

On a more fundamental and theoretical level, our model for membrane dynamics defines a novel universality class for phase separation in two dimensions with unique features. Indeed, standard models for phase separation were developed to study spinodal decomposition in alloys $\frac{20}{20}$, binary fluids 21 , reaction-diffusion, magnetism and wetting phenomena. They are generically described by the time-dependent Ginzburg-Landau (TDGL) equation -also called the Cahn-Allen equation 22 , or its conserved version the Cahn-Hilliard equation 23 , and give rise to power-law coars- 
ening (i.e. perpetual increase of the domain size) via curvaturedriven motion of domain walls. Here, we show that the dynamical equations governing confined membranes share similarities with the Swift-Hohenberg equation, however with a time-dependent tension that enforces membrane area conservation. Within this model, membrane adhesion domains exhibit a transition to coarsening controlled by the total excess area of the membrane.

The results reported here build on our previous study of membrane adhesion dynamics based on a one-dimensional (1D) model with bending rigidity, but without area conservation 24 . In 1D, we found that bending rigidity induces oscillatory interactions between domain walls. As a consequence of these oscillations, the dynamics freezes into a disordered or ordered profile depending on the permeability of the walls. In contrast to this behavior, we show here that in two-dimensions (2D) and without area conservation these oscillatory interactions between domain walls do not affect the coarsening behavior. Indeed, we recover standard coarsening with the same exponents as that of usual phase separation models (TDGL or Cahn-Hilliard). The study of such a model without area conservation can be motivated by the investigation of the coarsening dynamics of 2D systems at the Lifshitz-point, defined as the point where the prefactor of the gradient-squared term in the Landau free energy density vanishes 27 , and where higher-order bending-like squared-Laplacian terms come into play.

However, the present study focuses on the case of 2D membranes where local area conservation should be imposed. As discussed above, this leads to the presence of a time-dependent tension in the dynamical equations. The numerical solution of these equations reveals three regimes, hereafter denoted as A, B and C, depending on the excess area of the membrane.

For small excess area (regime A), the membrane freezes into a state with flat adhesion patches of finite size. This freezing can be understood as follows. Domain wall motion is driven by an effective positive wall tension, and acts so as to reduces the total domain wall length. Due to area conservation, domain wall length decrease implies an increase of the membrane excess area per unit length in domain walls. This increase induces a cancellation of the domain wall tension, leading to the arrest of domain wall motion. The dynamics in regime A can be analyzed within a simple model for the coupled dynamics of domain wall motion and of the nonlocal membrane tension. Since it corresponds to the cancellation of wall tension, the asymptotic value of the nonlocal tension is equal to the threshold tension for linear instability of domain walls in the SH equation.

For intermediate excess area (regime B), the membrane exhibits coarsening with a coexistence of flat and wrinkled domains. The wrinkle phase forms spontaneously when domain walls collide. The fraction of the system occupied by the wrinkle phase reaches a constant value at long times, which increases linearly with the excess area. Concurrently, the size of the flat and wrinkled domains increases indefinitely with time. Since the system reaches coexistence of wrinkles and flat domains, the asymptotic value of the nonlocal tension corresponds to the threshold for nonlinear stability of domain walls in the $\mathrm{SH}$ equation.

For larger excess area (regime $\mathrm{C}$ ), the wrinkle phase invades the whole system and the membrane freezes into a labyrinthine wrinkle pattern. The amplitude and wavelength of the wrinkles are analyzed within a simple sinusoidal ansatz, that reveals the presence of two different regimes within regime $\mathrm{C}$. For large excess area the amplitude of the wrinkles is fixed by the contact with the two walls, while wrinkles with smaller excess area exhibit a free amplitude smaller than the distance between the two walls.

We focus on the case with permeable walls, but also briefly report on the behavior of a simplified model for impermeable walls which exhibits similar dynamics. We also find unexpectedly that the number of adhesion domains is strictly conserved in all cases during the late stages of the dynamics due to the formation of robust localised structures which forbid the complete disappearance of the adhesion domains.

\section{Model Equations}

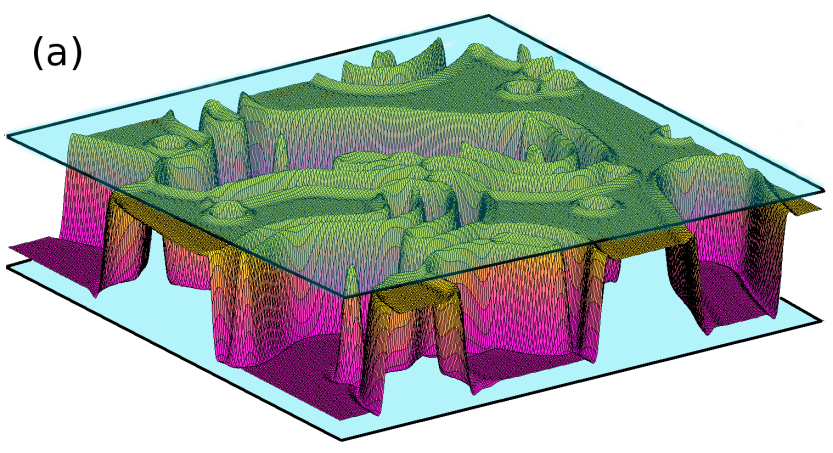

(b)

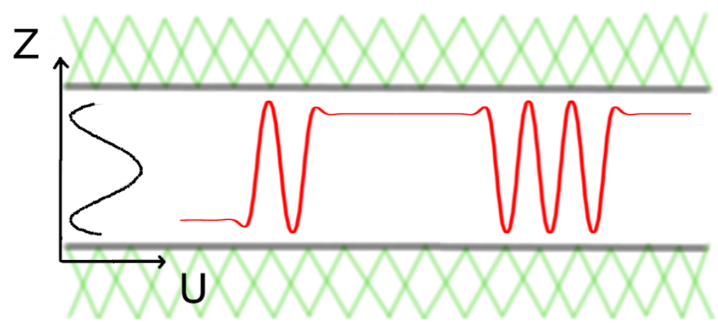

Fig. 1 (Color online) Membrane between two adhesive substrates. (a) 3D view; (b) 2D schematics: left black curve: double-well potential, right red curve: a slice of the membrane profile (profiles are obtained from simulation with $\Delta A^{*}=3.61 \cdot 10^{-2}$ at $T=8 \cdot 10^{5}$, corresponding to the last figure in regime $\mathrm{B} 2$ of Fig. 2 .

A schematic representation of a membrane of height $h(x, y, t)$ along $z$ confined between two parallel flat walls located at $z= \pm h_{0}$ is shown in Fig. 11 (a,b). The interaction between the membrane and the walls is modeled via a double-well potential $\mathscr{U}(h)$. Adding this interaction energy with the Helfrich bending energy, we obtain the total energy of the membrane as

$$
\mathscr{E}=\int d \mathscr{A}\left(\frac{\kappa}{2} \mathscr{C}^{2}+\mathscr{U}(h)\right),
$$

where $d \mathscr{A}$ denotes the infinitesimal area element of the membrane, $\mathscr{C}$ denotes the membrane local curvature and $\kappa$ denotes the bending rigidity. 
In addition, the local conservation of the membrane area can be expressed as a conservation of the membrane area density $\rho(x, y, t)=\left[1+\left(\nabla_{x y} h\right)^{2}\right]^{1 / 2}$ :

$$
\partial_{t} \rho+\nabla_{x y} \cdot\left(\rho \mathbf{v}_{x y}\right)=0,
$$

where $\mathbf{v}_{x y}$ is the membrane velocity parallel to the walls, $\nabla_{x y}$ is the gradient in the $(\mathrm{x}, \mathrm{y})$ plane. In order to enforce this constraint, we make use of a local space-dependent and time-dependent Lagrange multiplier $\sigma(x, y, t)$ (which can be interpreted as a local membrane tension $28 \mid 29$ ). The membrane energy is thus generalised as

$$
\mathscr{X}=\int d \mathscr{A}\left(\frac{\kappa}{2} \mathscr{C}^{2}+\mathscr{U}(h)+\sigma\right) .
$$

The local tension $\sigma(x, y, t)$ leads to an additional contribution to membrane forces, which is constrained to obey Eq. (2) at all times. Such a constraint allows one to determine $\sigma$.

Since all the phenomena described here occur at small scales, we consider the Stokes regime where inertia is negligible. Hence, membrane forces $\mathbf{f}$ resulting from energy variations and inextensibility have to balance viscous forces exerted by the surrounding liquid on the membrane:

$$
\mathbf{f}=\left(\left.\mathbf{s}_{+}\right|_{z=h(x, y, t)}-\left.\mathbf{s}_{-}\right|_{z=h(x, y, t)}\right) \cdot \mathbf{n}
$$

where $\mathbf{s}$ is the stress tensor in the liquid, \pm denotes the liquid above or below the membrane, and $\mathbf{n}$ is the normal to the membrane. The liquid with velocity $\mathbf{v}_{ \pm}$obeys the incompressible Stokes equations, with $\nabla \cdot \mathbf{v}=0$ and $\nabla \cdot \mathbf{s}=0$, where $\mathbf{s}_{i j}=\mu\left(\partial_{i} v_{j}+\partial_{j} v_{i}\right)-p \delta_{j i}$ where $\mu$ is the fluid viscosity and $p$ its pressure. In addition, we assume no-slip $30 \mid 31$ and impermeability $32-34$ at the membrane, leading to

$$
\left.\mathbf{v}_{+}\right|_{z=h(x, y, t)}=\left.\mathbf{v}_{-}\right|_{z=h(x, y, t)} .
$$

In order to account for the permeability of the walls, we impose

$$
\left.v_{z}\right|_{z= \pm h_{0}}= \pm v\left(p_{ \pm}-p_{\text {ext }}\right),
$$

where $p_{\text {ext }}$ is a reference constant pressure, and $v$ is the wall permeability. Finally, we assume a no-slip condition at the walls $\left.\mathbf{v}_{x y \pm}\right|_{z= \pm h_{0}}=0$.

\section{Lubrication limit: permeable walls}

We apply the lubrication limit where $\left|\nabla_{x y} h\right|$ is small. In this limit, the hydrodynamic flow above and below the membrane are simple Poiseuille flows parallel to the walls. Using the boundary conditions presented in the previous section, the hydrodynamic flows can be obtained explicitly. We then obtain dynamical equations for the membrane profile using Eq. (5).

The derivation of the evolution equation for $h$ in this limit actually follows the same steps as the simpler case of a one-dimensional membrane without area conservation reported in Ref. 24. The main difference comes from the constraint of membrane incompressibility. The details of the calculations and the general result are reported in Appendix A We will mainly focus on the limit of large normalised permeability, $\bar{v} \gg 1$, with

$$
\bar{v}=\frac{12 \mu \nu \kappa^{1 / 2}}{h_{0}^{2} \mathscr{U}_{0}^{1 / 2}} .
$$

where $\mathscr{U}_{0}$ is the amplitude of the interaction potential.

In physical systems, the value of $\bar{v}$ depends crucially on the permeability $v$ of the substrate. One possibility to evaluate $\bar{v}$ is to use Darcy's law for porous media, using $v \sim a_{0}^{2} /\left(\mu h_{w}\right)$, where $a_{0}$ is the scale of the pores and $h_{w}$ the thickness of the wall. This suggests $\bar{v} \sim 12 a_{0}^{2} \kappa^{1 / 2} /\left(h_{0}^{2} h_{w} \mathscr{U}_{0}^{1 / 2}\right)$. In the case of scaffolded actin cytoskeleton, the typical pore size is $a_{0} \sim 10^{-7} \mathrm{~m}$ and the thickness $h_{w} \sim 10^{-6} \mathrm{~m}$. Moreover, assuming that the energy-scale of the potential is dictated by the cytoskeleton-lipid membrane adhesion energy, we find $36 \mathscr{U}_{0} \sim 10^{-5} \mathrm{~J}^{-m^{-2}}$. Using ${ }^{3738} \kappa \sim 10^{-19} \mathrm{~J}$ and $h_{0} \sim 10^{-8} \mathrm{~m}$, we find $\bar{v} \sim 10^{2}$. For collagen, a very common extracellular matrix, the typical pore size is $10^{-6} \mathrm{~m}$ and thickness is $10^{-4} \mathrm{~m}^{39}$ which gives same order of magnitude for $\bar{v}$.

However, if the substrate is covered by another lipid membrane, the permeability of the substrate becomes very small 32,34 . An increase of about 1 atm of osmotic pressure induces a speed of about $10^{-4} \mathrm{~m} . \mathrm{s}^{-1}$ for the water across a lipid membrane 32 . 34 . This means that from Eq. (6) $v \sim 10^{-9} \mathrm{~m}^{2} \cdot \mathrm{s} . \mathrm{kg}^{-1}$. Then using water viscosity, and the same values as above $\kappa \sim 10^{-19} \mathrm{~J}, h_{0} \sim 10^{-8} \mathrm{~m}$ and $\mathscr{U}_{0} \sim 10^{-5} \mathrm{~J} . \mathrm{m}^{-2}$, we find $\bar{v} \sim 10^{-2}$. We will briefly discuss the case of small permeabilities in the end of the paper.

In the limit of strongly permeable walls $\bar{v} \gg 1$, the dynamical equation reads

$$
\begin{gathered}
\partial_{t} h=\frac{v}{2}\left(-\kappa \Delta^{2} h+\sigma_{0} \Delta h-\mathscr{U}^{\prime}(h)\right), \\
\sigma_{0}=\frac{\iint d x d y\left(\kappa \Delta^{2} h+\mathscr{U}^{\prime}(h)\right) \Delta h}{\iint d x d y(\Delta h)^{2}} .
\end{gathered}
$$

Here, $\Delta$ denotes the Laplacian operator in planar coordinates $(x, y)$. One important and non-trivial result emerging from the lubrication limit is that, to leading order, the local Lagrange multiplier $\sigma(x, y, t)$ is constant in space, with a value $\sigma_{0}(t)$, thereby leading to a nonlocal area conservation constraint.

The evolution equations Eqs. (89) decrease the energy, here written in the small slope limit as

$$
\mathscr{E}=\int d \mathscr{A}\left(\frac{\kappa}{2}\left(\nabla^{2} h\right)^{2}+\mathscr{U}(h)\right) .
$$

Indeed, as shown in Appendix B using the Schwarz inequality, we always have $\partial_{t} \mathscr{E} \leq 0$.

\section{Normalization and numerical methods}

In order to discuss the dynamical behavior of Eqs. (89) and to solve the equations numerically, we normalise space and time, leading to

$$
\begin{array}{r}
\partial_{T} H=-\Delta^{2} H+\Sigma \Delta H-U^{\prime}(H), \\
\Sigma=\frac{\iint d X d Y\left(\Delta^{2} H+U^{\prime}(h)\right) \Delta H}{\iint d X d Y(\Delta H)^{2}} .
\end{array}
$$


where $U(h)=\mathscr{U}(H) / \mathscr{U}_{0}, T=v \mathscr{U}_{0} t /\left(2 h_{0}^{2}\right), H=h / h_{0}, X=x / \mathscr{L}_{0}$, $Y=y / \mathscr{L}_{0}$, with $\mathscr{L}_{0}=\kappa^{1 / 4} h_{0}^{1 / 2} / \mathscr{U}_{0}^{1 / 4}$, and $\sigma_{0}=\Sigma\left(\mathscr{U}_{0} \kappa\right)^{1 / 2} / h_{0}$. Using the numerical values of the previous section, we obtain the order of magnitude of tensions $\left(\mathscr{U}_{0} \kappa\right)^{1 / 2} / h_{0} \sim 10^{-4} \mathrm{~J}_{\mathrm{m}}{ }^{-2}$, and the typical lengthscale parallel to the membrane $\mathscr{L}_{0} \sim 30 \mathrm{~nm}$. The small slope limit amounts to considering $h_{0} / \mathscr{L}_{0} \approx 0.3$ is small as compared to 1 . Although these slopes are not very small, the lubrication limit is known to provide a qualitatively good and robust description of the physical behavior for moderate slopes 40 . In addition, smaller adhesion energies, e.g., using $\mathscr{U}_{0} \sim 2 \times 10^{-6} \mathrm{Jm}^{-2}$ as suggested by Ref. $\frac{16}{16}$, or $\mathscr{U}_{0} \sim 1.4 \times 10^{-6} \mathrm{Jm}^{-2}$ in Ref. $\frac{13}{13}$, lead to even smaller slopes. In general, the adhesion energy, on which the validity of this limit depends crucially, is system-dependent and varies strongly with the type and the density of binders. Considering the different case of physical adhesion with a porous solid substrate, a crude approximation consists in multiplying the physical adhesion potential of Ref. ${ }^{41}$ _based on Van der Waals and hydration interactions, with the solid fraction $\vartheta$. Assuming for example $\vartheta \approx 0.3$, we then find a value $\mathscr{U}_{0} \approx 1.5 \times 10^{-6} \mathrm{Jm}^{-2}$ similar to those reported above. Summarizing this discussion, we expect in most cases $\mathscr{L}_{0} \sim 30$ to $50 \mathrm{~nm}$ for nano-confinement with $h_{0} \sim 10 \mathrm{~nm}$.

We choose a specific form of the double-well adhesion potential

$$
U(H)=\frac{1}{4}\left(H_{m}^{2}-H^{2}\right)^{2}+U_{d}
$$

where

$$
U_{d}=U_{1}\left[\mathrm{e}^{-(1-H) / d}+\mathrm{e}^{-(1+H) / d}\right]
$$

is a short-range repulsion, the aim of which is to avoid collision of the membrane with the walls. In the simulations, we have chosen $H_{m}=0.7, d=0.01$. Assuming that $h_{0} \sim 10 \mathrm{~nm}$, the position of the minimum of the potential corresponds to a distance to the substrate $h_{0}\left(1-H_{m}\right) \sim 3 \mathrm{~nm}$, which is similar to those reported in the literature for physical interactions ${ }^{41}$, or binders 13 . During the simulations, we usually assumed that $U_{1}=1$. However, we used $U_{1}=0$ to accelerate long simulations in the regimes where the membrane did not approach the walls.

The dynamics is integrated by means of a first-order exponential-time-differencing method (ETD1) in Fourier space ${ }^{42}$ with space and time discretization bins $d X=0.4$, and $d T=0.15$. We have modified the integration scheme in order to conserve exactly the excess area

$$
\Delta A=\frac{1}{2} \iint d X d Y(\nabla H)^{2} .
$$

Our scheme amounts to choosing a specific discretization of the expression of the tension $\Sigma$ in Eq. (12) in order to enforce exact area conservation. The details of this scheme are presented in Appendix C.1.

The simulation box sizes where usually $L_{X} \times L_{Y}=400 \times 400$, or $800 \times 800$ corresponding to physical sizes from 10 to $40 \mu \mathrm{m}$.

\section{Area-preserving vs tensionless mem- branes}

We start with noisy initial conditions (details on these conditions are described in Appendix C.2). Three different regimes are obtained depending on the excess area density

$$
\Delta A^{*}=\frac{\Delta A}{A_{\text {syst }}} .
$$

where $A_{\text {syst }}$ is the system size in the $(X, Y)$ plane (if the simulations are performed in a rectangular box of size $L_{X} \times L_{Y}$, we have $A_{\text {syst }}=L_{X} L_{Y}$ ). For increasing excess area density, we first find a regime with frozen flat domains, then a regime with coarsening and with coexistence between flat domains and wrinkles, and finally a regime with a disordered pattern of frozen wrinkles. These three regimes will be denoted as regimes A, B and C in the following. Some snapshots of these evolutions are shown in Fig. 2

Since frozen states were also observed in a one-dimensional model without area conservation in Ref. 24 , we wish to investigate the behavior of the model without the constraint of area conservation as a preamble to the analysis of the full model. This is done by simulating Eq. (11) with $\Sigma=0$. The resulting equation was called the fourth order Time-dependent Ginzburg-Landau equation (TDGL4) in Ref. 24 . Such an equation corresponds to a system that would exhibit bending rigidity, but for which the extension of the area of the interface would occur at no cost (physically corresponding to dynamics at a Lifshitz point).

Frozen disordered states obtained in simulations of TDGL4 in $1 \mathrm{D}^{24}$ can be seen as a consequence of trapping of domain-walls (called kinks in 1D) into their mutual oscillatory interactions. These oscillations, which can be traced back to the oscillatory tails of the domain wall profiles, are still present in 2D. The first oscillation can indeed be observed in the vicinity of all domain walls in Fig. 1. However, the TDGL4 dynamics in 2D, shown in Fig. 3(a), actually leads to a simple coarsening behavior with flat adhesion patches, the size of which grows like $t^{1 / 2}$. This is identical to the $2 \mathrm{D}$ behavior of the TDGL equation. Such a coarsening behavior is usually interpreted as a consequence of motion of domain walls driven by their curvature. Thus, the coarsening behavior of TDGL4 suggests that, in absence of area-conservation constraint, motion by curvature of domain walls dominates over oscillatory interactions.

As mentioned above, membranes with area conservation exhibit strikingly different dynamics. Hereafter, we discuss the three dynamical regimes arising in membranes with area conservation.

\section{Small excess area}

Regime A corresponds to simulations of Eqs. (11) 12 at small excess area. In this regime, flat adhesion domains expand initially (Fig. 2A, $T=15$ ), and the tension $\Sigma$ decreases to negative values. Later, these domains freeze $2 \mathrm{~A}, T=10^{6}$ ), and $\Sigma$ reaches a constant negative value independent of initial conditions and of the imposed excess area. The evolution of the domain size $\lambda_{\text {flat }}$ and of the tension are shown in Fig. 4(a,c).

When $\Sigma$ is constant in time, our dynamical equation (11) is identical to the much-studied Swift-Hohenberg ( $\mathrm{SH}$ ) equation 43,47 


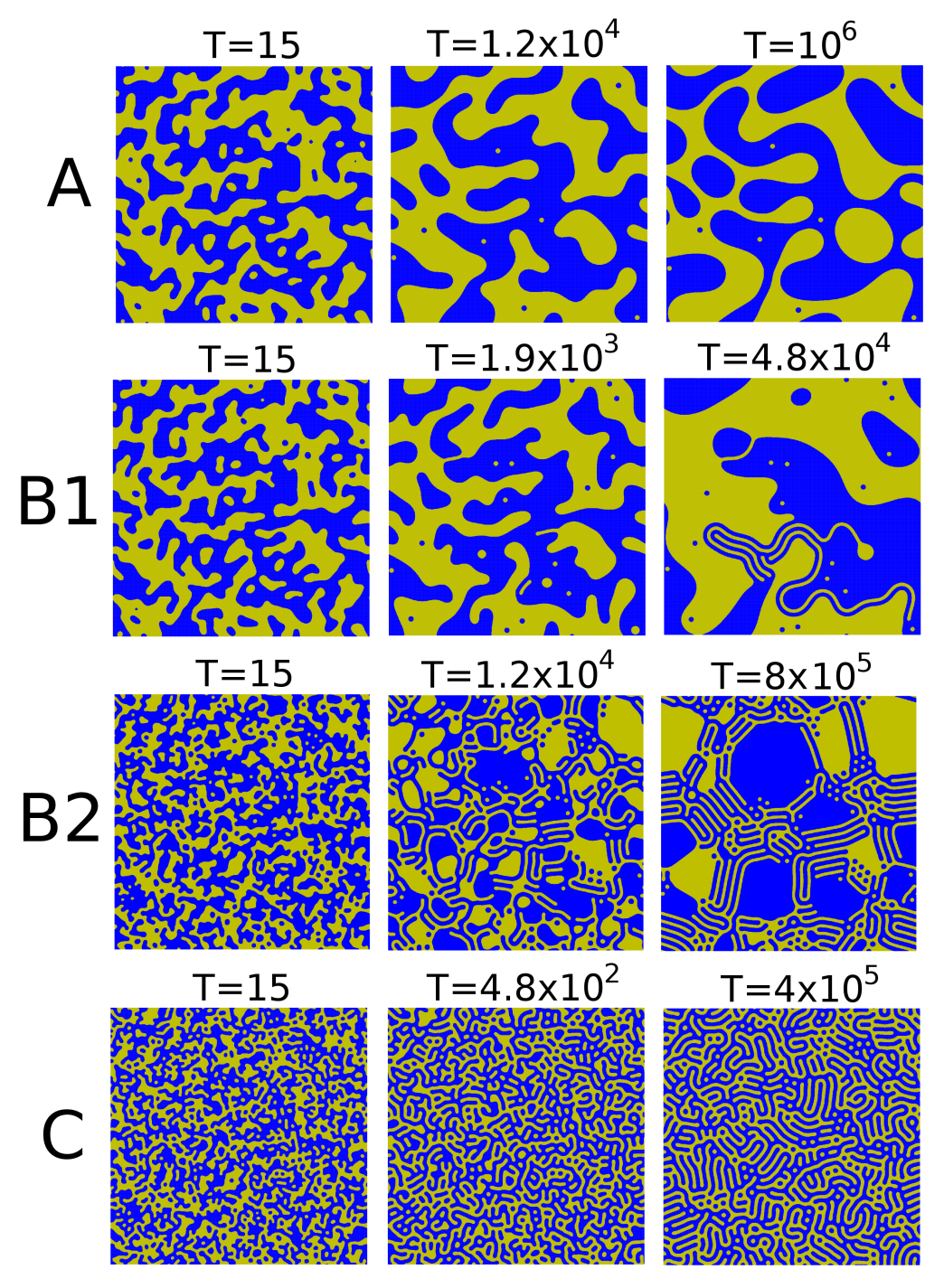

Fig. 2 (Color online) Membrane adhesion dynamics for various excess areas. Yellow: adhesion patches on the upper wall. Dark blue: adhesion patches on the lower wall. Regime $A$ with freezing of flat domains for small excess area : $\Delta A^{*}=0.88 \times 10^{-2}$. Regime $\mathrm{B}$ with coexistence of the flat-domain phase and wrinkle phase with coarsening for intermediate excess area: B1 with $\Delta A^{*}=1.08 \times 10^{-2}$, and $\mathrm{B} 2$ with $\Delta A^{*}=3.61 \times 10^{-2}$. Regime C with frozen wrinkles for larger excess area $\Delta A^{*}=5.68 \times 10^{-2}$. System size $L \times L=400 \times 400$.

(neglecting the contribution $U_{d}$ to the potential). Hence, the steady-states of our model are steady-states of the $\mathrm{SH}$ equation. However, the stability of these steady-states can be different. We observe that the value towards which $\Sigma$ converges in our simulations is close to the limit of linear stability of flat domain walls with respect to transverse perturbation in the SH model at $\Sigma_{c} \approx-1.0226 H_{m}$ as reported by Hagberg et al.47. Such a limit of stability is associated with the cancellation of the energy of domain walls in the $\mathrm{SH}$ equation.

In order to explore the consequence of this cancellation, we need to relate the energy in the two models. In normalised form, they read:

$$
\begin{aligned}
& E=\int d A\left\{\frac{1}{2}(\Delta H)^{2}+U(H)\right\}, \\
& \Xi=\int d A\left\{\frac{1}{2}(\Delta H)^{2}+U(H)+\frac{\Sigma}{2}(\nabla H)^{2}\right\} .
\end{aligned}
$$

The energies $e_{D W}$ and $\xi_{D W}$ per unit length of a straight and isolated domain wall in our model and in the SH model therefore read

$$
\begin{aligned}
& e_{D W}=\int d \zeta\left\{\frac{1}{2}\left(\partial_{\zeta \zeta} H\right)^{2}+U(H)\right\}, \\
& \xi_{D W}=\int d \zeta\left\{\frac{1}{2}\left(\partial_{\zeta \zeta} H\right)^{2}+U(H)+\frac{\Sigma}{2}\left(\partial_{\zeta} H\right)^{2}\right\},
\end{aligned}
$$



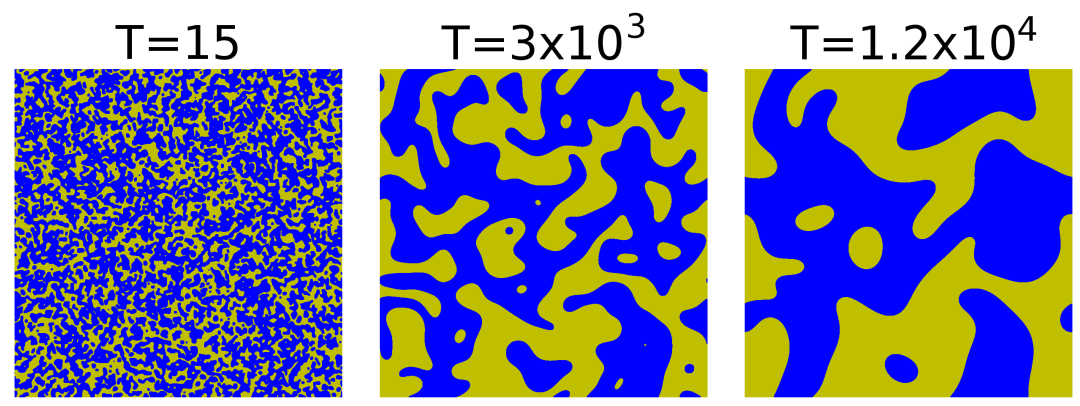

(a)
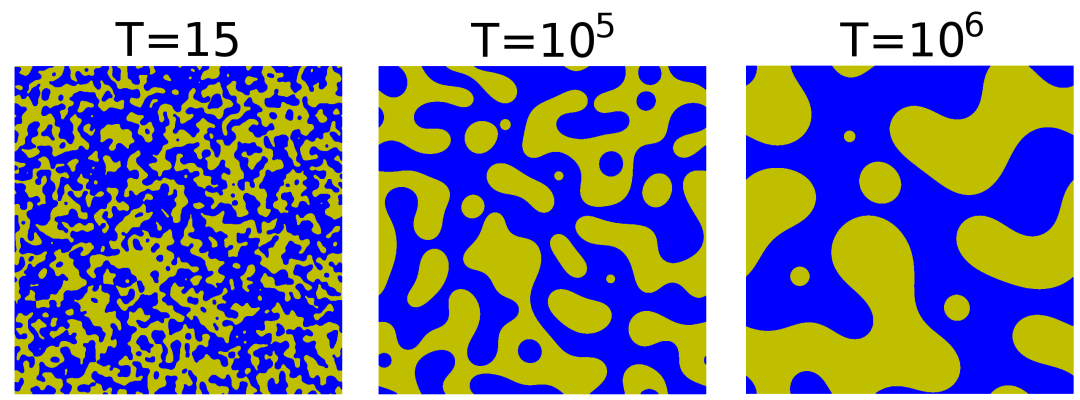

(b)

Fig. 3 (Color online) (a) TDGL4 (Eq. 8 with $\Sigma=0$ ), $L=1600, L_{D W} \sim T^{1 / 2}$. (b) CH4 (constant mobility) (Eq. 35a with $M(H)=1$ and $\Sigma=0$ ), $L=800$, $L_{D W} \sim T^{1 / 3}$.

where $\zeta$ is the coordinate orthogonal to the domain wall. Assuming that the whole excess area is stored in domain walls, we have

$$
\Delta A \approx L_{D W} \alpha_{D W}
$$

where $L_{D W}$ is the length of domain walls in the system, and

$$
\alpha_{D W}=\frac{1}{2} \int d \zeta\left(\partial_{\zeta} H\right)^{2}
$$

is the excess area per unit domain wall length. Combining Eqs. $\left.\begin{array}{l|l|l|l|l|}\hline 19 & 20 & 21\end{array}\right)$, we find

$$
\xi_{D W}=e_{D W}+\frac{\Sigma \Delta A}{L_{D W}}
$$

Since $\xi_{D W}=0$ when $\Sigma=\Sigma_{c}$, we obtain an expression for the size of the domains in the frozen state of our model

$$
\bar{\lambda}_{\text {flat }}=\frac{A_{\text {syst }}}{L_{D W}}=\frac{e_{D W}^{c}}{-\Sigma_{c} \Delta A^{*}},
$$

where $e_{D W}^{c}$ is the value of $e_{D W}$ for $\Sigma=\Sigma_{c}$.

Using independent $1 \mathrm{D}$ simulations of the $\mathrm{SH}$ equation in a periodic box with two opposite domain walls, we have determined $e_{D W}$ as a function of $\Sigma$ in steady-state, as seen in Fig. 5. In particular, we find $e_{D W}^{c} \approx 0.2315$. Inserting this value in Eq. (24), we obtain a prediction of $\bar{\lambda}_{\text {flat }}$ in good agreement with numerical results, as shown by the left red curve on Fig. 6

We now discuss why the tension converges to the special value $\Sigma_{c}$. In order to investigate this point, we make use of an effective model for domain wall motion. The derivations are inspired from that of Ref. 25 , and some details are reported in Appendix D. As expected, we find that domain wall motion can be driven either by wall-wall interactions or by curvature, and the local normal velocity of the wall obeys

$$
V_{n}=-\frac{1}{\alpha_{D W}}\left(\left[U_{0}\right]_{-}^{+}+K \xi_{D W}\right)
$$

where $K$ is the local domain wall curvature, and $\left[U_{0}\right]_{-}^{+}$is an interaction term (see Appendix $D$ for its detailed expression). As discussed above, interactions between two domain walls are known to be oscillatory 25 , and to decay exponentially. They become negligibly small when wall-wall distances exceed a few domain wall thicknesses.

In regime A, domain walls are seen to be far apart, and as a consequence, domain wall motion should be mainly driven by curvature. Such curvature-driven domain wall motion leads to changes in the system configuration that reduce the total length $L_{D W}$ of the domain walls when $\xi_{D W}>0$, i.e. when $\Sigma>\Sigma_{c}$ from Fig. 5. Indeed, using the geometric relation $\partial_{t} L_{D W}=\int d \ell_{W D} K V_{n}$, and inserting the expression of $V_{n}$ from Eq. (25) neglecting the interaction term, we find $\partial_{t} L_{D W}=-\left(\xi_{D W} / \alpha_{D W}\right) \int d \ell_{W D} K^{2}<0$. Since the total excess area $\approx L_{D W} \alpha_{D W}$ is conserved, this decrease of $L_{D W}$ leads to an increase of the excess area $\alpha_{D W}$ stored per unit length in domain walls. Such a change in $\alpha_{D W}$ is intuitively associated to a decrease of the tension $\Sigma$ toward more negative values. Indeed, we expect $\partial_{\Sigma} \alpha_{D W} \leq 0$, i.e., more positive tensions correspond to pulling the membrane out from the domain walls which decrease the excess area inside the domain wall, while more negative tensions corre- 


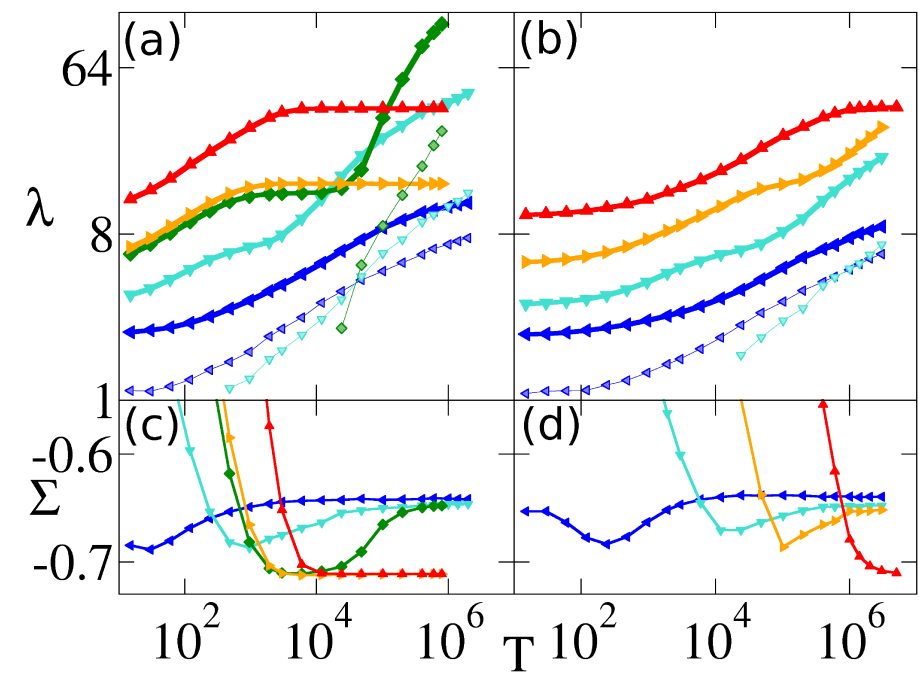

Fig. 4 (Color online) Time-evolution of the lengthscale $\lambda$ and the tension $\Sigma$. Size of the simulation box $L=800$. (a) and (c): results in the limit of large wall permeability. (b) and (d): results for impermeable walls. In (a) and (b), thick lines correspond to $\lambda_{\text {flat }}$, and thin lines to $\lambda_{w r}$. Excess area:

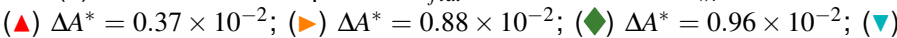
$\Delta A^{*}=1.8 \times 10^{-2} ;(\triangleleft) \Delta A^{*}=3.61 \times 10^{-2}$.

spond to pushing towards the domain walls leading to an increase of excess area. The inequality $\partial_{\Sigma} \alpha_{D W} \leq 0$ is confirmed by $1 \mathrm{D}$ simulations of the SH equation in steady-state reported in Fig. 5

As a summary, curvature-driven wall motion leads to an increase of $\alpha_{D W}$ accompanied by a decrease of the tension $\Sigma$. This decrease is governed by the equation

$$
\partial_{T} \Sigma=\frac{\xi_{D W} \int d \ell_{D W} K^{2}+\int d \ell_{D W}\left[U_{0}\right]_{-}^{+} K}{L_{D W} \partial_{\Sigma} \alpha_{D W}} .
$$

where $\ell_{D W}$ is the arclength along the domain walls, and the integration runs over all domain walls. This relation is a consequence of area conservation, and its derivation is reported in Appendix D. Once again, we neglect the interaction terms proportional to $\left[U_{0}\right]_{-}^{+}$. As seen in Fig. $5 \xi_{D W}$ is an increasing function of $\Sigma$. Since $\partial_{T} \Sigma$ is proportional to $\xi_{D W}$, and recalling that $\partial_{\Sigma} \alpha_{D W} \leq 0$, Eq. 26] shows that $\Sigma$ will decrease up to the point where $\xi_{D W}=0$, where it reaches the constant $\Sigma_{c}$. Since $\xi_{D W} \rightarrow 0$, the motion of domain walls freezes from Eq. (25).

As already mentioned above, this discussion is based on the assumption that interactions between domain walls are weak. Since interactions decrease exponentially with the distance, such an assumption should be valid in the limit where the distance between domain walls is large enough. However, as $\Delta A^{*}$ increases, this distance decreases, and interactions between walls should become relevant. Indeed, a different regime, discussed in the next section and hereafter denoted as regime $\mathrm{B}$ appears for larger $\Delta A^{*}$.

\section{Intermediate excess area}

As announced in the previous sections, a different regime is observed for larger excess area. This regime, denoted as regime B is

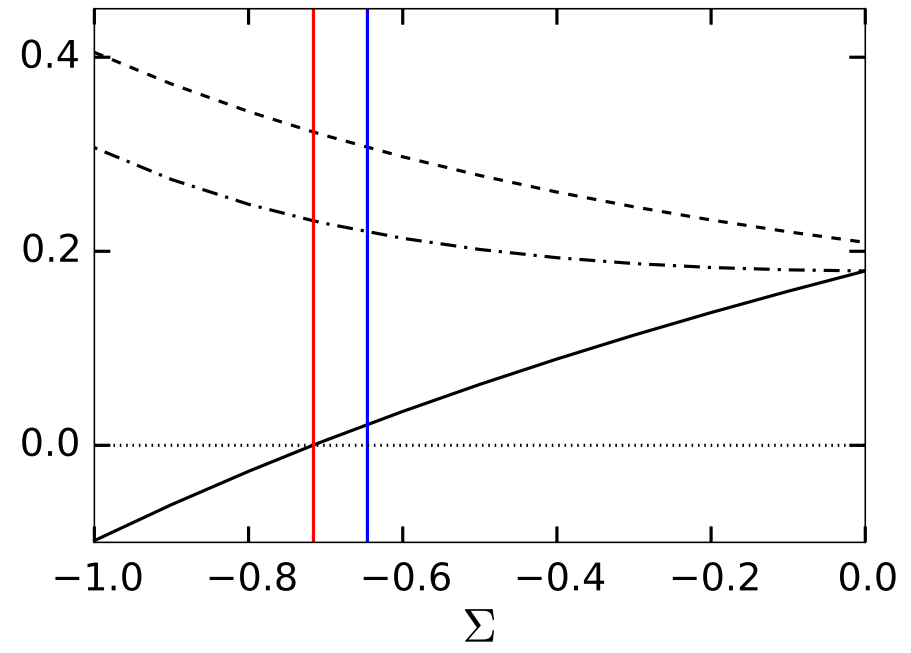

Fig. 5 (Color online) Domain wall energy and excess area. Continuous curve: $\xi_{D W}$. Dashed curve: $\alpha_{D W}$. Dotted-dashed curve: $e_{D W}$. The vertical lines indicate $\Sigma_{c}$ (left red), and $\Sigma_{n l}$ (right blue).

found for $\Delta A_{c}^{*}<\Delta A^{*}<\Delta A_{n l}^{*}$, with

$$
\begin{aligned}
\Delta A_{c}^{*} & =(0.93 \pm 0.03) \times 10^{-2} \\
\Delta A_{n l}^{*} & =(5.53 \pm 0.15) \times 10^{-2}
\end{aligned}
$$

The errors on $\Delta A_{c}^{*}$ and $\Delta A_{n l}^{*}$ are based on the difference between the closest upper and lower bounds for the transition obtained by long-time simulations in a system of size $L=L_{X}=L_{Y}=800$.

In regime $B$, both flat and wrinkled domains coexist and expand perpetually (see regimes B1 and B2 in Figs. 2). Numerical results for the dynamics of the tension and of the size of the domains are shown in Figs. 4(a) and 4(c), with the symbols $(\boldsymbol{\Delta}),(\vee),(\nabla),(\nabla)$ and $(\triangleleft)$ in the order of increasing $\Delta A^{*}$. Regime B corresponds to the symbols $(\nabla),(\nabla)$ and $(\triangleleft)$. The typical sizes of flat domains $\lambda_{\text {flat }}$ and the size of wrinkle domains $\lambda_{w r}$ both increase with time. The method for extracting these lengths from simulation data is discussed in Appendix C.4. The growth rates of $\lambda_{\text {flat }}$ and $\lambda_{w r}$ decrease with increasing $\Delta A^{*}$. However, no universal exponent related to this coarsening process could be found within our simulations. In addition, we observe that the fraction $\phi_{w r}$ of the system covered with the wrinkle phase reaches a constant at long times, as shown in Fig. 8(a).

As seen in Fig. $4(\mathrm{c})$, the tension in regime B first decreases to a minimum value close to or larger than $\Sigma_{c}$, and then increases to another constant asymptotic value. This value, hereafter denoted as $\Sigma_{n l}$, corresponds to a point of coexistence of flat and wrinkled states in our system. Following the same procedure as for the definition of generalised thermodynamic potentials, coexistence between two states correspond to the equality of Legendretransformed energy $\Xi$ with respect to the Lagrange multiplier $\Sigma$ conjugate to the fixed quantity $\Delta A$. Coexistence therefore corresponds to the point where the $\Xi$-energy densities of the flat and wrinkled states are equal: $\Xi_{F D} / A_{F D}=\Xi_{w r} / A_{w r}$, where the indexes "FD" and "wr" respectively correspond to the flat domains regions 


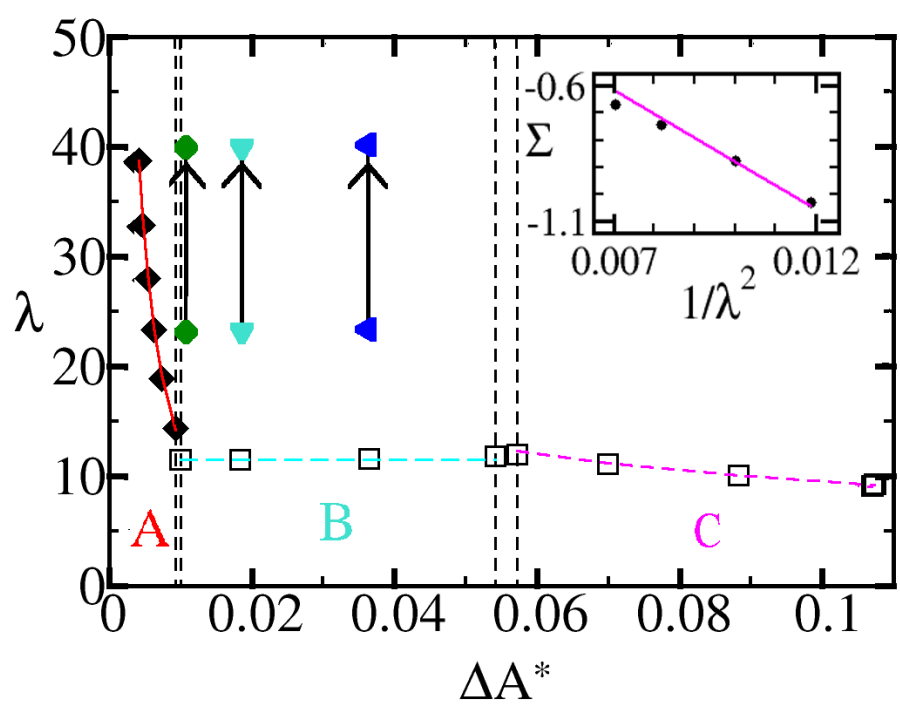

Fig. 6 (Color online) Typical length scale $\lambda$ at long times as a function of $\Delta A^{*}$ for $L=L_{X}=L_{Y}=800$. Symbols: $\nabla$ for $\lambda_{\text {flat }}$, and $\square$ for $\lambda_{1 w r}$. The critical values of the normalised excess area $\Delta A_{c}^{*}$ and $\Delta A_{n l}^{*}$ are indicated with dashed black lines (these lines are doubled to indicated the accuracy of our measurement of the transition). Thin red curve and thin magenta curve: analytical results. Inset: linear dependence of $\Sigma$ on $1 / \lambda^{2}$. Symbols for simulation results $\bullet$. Magenta line: $\Sigma=-88 / \lambda^{2}$.

and wrinkles regions.

Note that the energy $\Xi_{F D}$ a priori contains not only the contribution of flat domains with $H= \pm H_{m}$, but also that of domain walls inside this region. However, the density of domain walls is low, and we shall only consider the contribution of flat domains, for which $\Xi_{F D}=0$ (such a cancellation relies on the assumption that the minimum $H_{m}$ is far enough form the walls for the short-range potential $U_{d}$ to be negligible at $H=H_{m}$, i.e., $\left.1-H_{m} \ll d\right)$. Following the same lines, the energy within the wrinkle phase contains a contribution due to wrinkle bending and wrinkle defects, which is also neglected. We therefore end up with a simpler condition of coexistence $\Xi_{w r}=0$, calculated for a periodic phase of parallel rolls. This is exactly the condition of nonlinear stability of domain walls with respect to the formation of a wrinkle phase in $\mathrm{SH}$ as discussed in Ref. $\frac{47}{4}$, leading to $\Sigma_{n l} \approx-0.9225 H_{m}$ in agreement with our simulation results.

Using this concept of coexistence, together with the observation of the variation of the tension with time, we propose a scenario in three stages for regime B. First, the dynamics looks like that of regime A, and the evolution is dominated by domain wall motion, leading to a decrease of $\Sigma$ toward $\Sigma_{c}<\Sigma_{n l}$. Second, once $\Sigma<\Sigma_{n l}$, the flat states become unstable with respect to the formation of the wrinkle state. In simulations at low excess area, i.e., close to regime A, this instability usually occurs as follows: two domain walls collide and form an isolated wrinkle, which grows by zipping more domain walls. This is consistent with the inequality $\xi_{1 w r}<2 \xi_{D W}$, where $\xi_{1 w r}$ is the $\Xi$-energy per unit length of a straight and isolated wrinkle, which can be checked on Fig. 7. For larger excess area, the separation between the two initial stages is less clear since many domain walls are already close to each

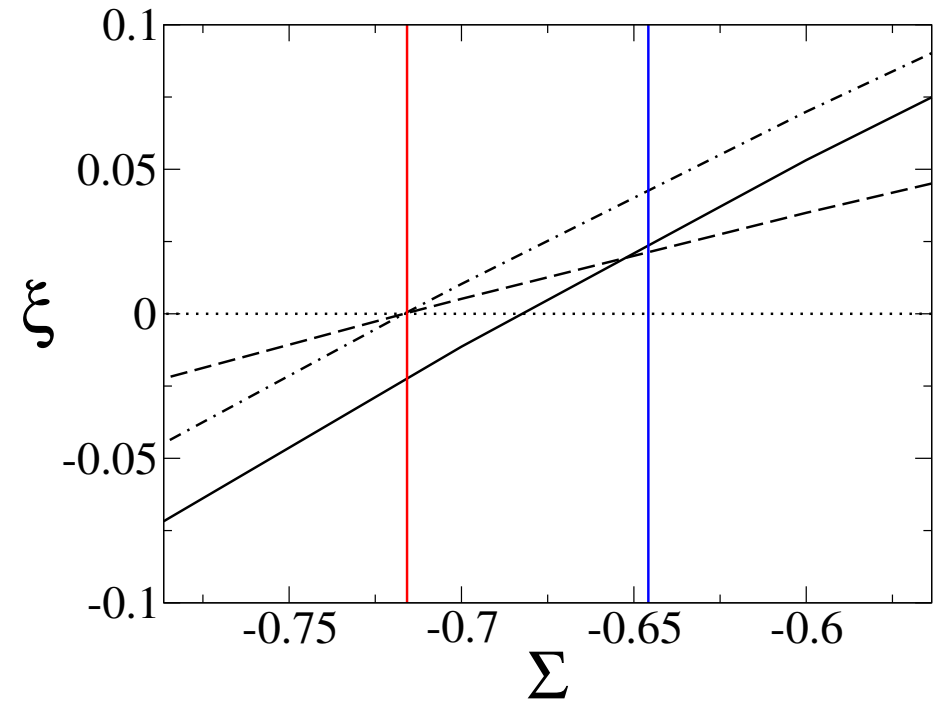

Fig. 7 (Color online) Comparison of the energies of an isolated wrinkle, and of two domain walls. Left red vertical line: $\Sigma_{c}$. Right blue vertical line: $\Sigma_{n l}$. Solid line: $\xi_{1 w r}$. Dashed line: $\xi_{D W}$. Dashed-dotted line: $2 \xi_{D W}$.

other initially. Finally, in the third stage, coarsening occurs with coexistence of wrinkle domains and flat domains. It is tempting to speculate that the coarsening process is driven by the decrease of the length of frontiers between the coexisting wrinkled state and the flat state. However, annihilation of defects within the wrinkled state and motion of simple domain walls between flat domains could also play a role.

When the excess area is increased, the fraction of the system covered by wrinkles increases. When the excess area exceeds a threshold value, the full system is covered by wrinkles, and a different regime, denoted as regime $\mathrm{C}$ is found.

Some analysis of the fraction $\phi_{w r}$ of the system occupied by wrinkles in regime $B$ is possible assuming once again that the contribution of defects such as domain walls in the flat phase and defects in the wrinkle phase are negligible. The wrinkle state is then composed of parallel rolls of wavelength $\lambda_{1 \text { roll }}^{n l}$ where the superscript $n l$ indicates that this quantity is evaluated for $\Sigma=\Sigma_{n l}$. We define wrinkle length $L_{w r}$, i.e., the total wrinkle length summed over all wrinkles (formally, this can be defined as, e.g., the total length of all the lines of local maximums of the wrinkles in the whole system). Assuming that all the excess area is stored in the wrinkle phase, one has $\Delta A=\alpha_{1 \text { roll }}^{n l} L_{w r}$. The area covered by wrinkles then reads $A_{w r}=L_{w r} \lambda_{1 \text { roll }}^{n l}$. Combining these relations, we find that the fraction $\phi_{w r}=A_{w r} / A_{s y s t}$ of the system covered by the wrinkle state is proportional to the normalised excess area $\Delta A^{*}$ :

$$
\phi_{w r}=\Delta A^{*} \frac{\lambda_{1 \text { roll }}^{n l}}{\alpha_{1 \text { roll }}^{n l}} .
$$

In order to determine the quantities appearing in the right hand side of Eq. (29), we simulated the SH equation with $\Sigma=\Sigma_{n l}$ in 1D in a box of size $L=800$. We found that a stable wrinkle profile is reached with 66 or 67 wavelengths. Taking the average of these values, we obtain $\lambda_{1 \text { roll }}^{n l}=12.03 \pm 0.09, \alpha_{1 \text { roll }}^{n l}=0.7006 \pm 0.0042$, and 


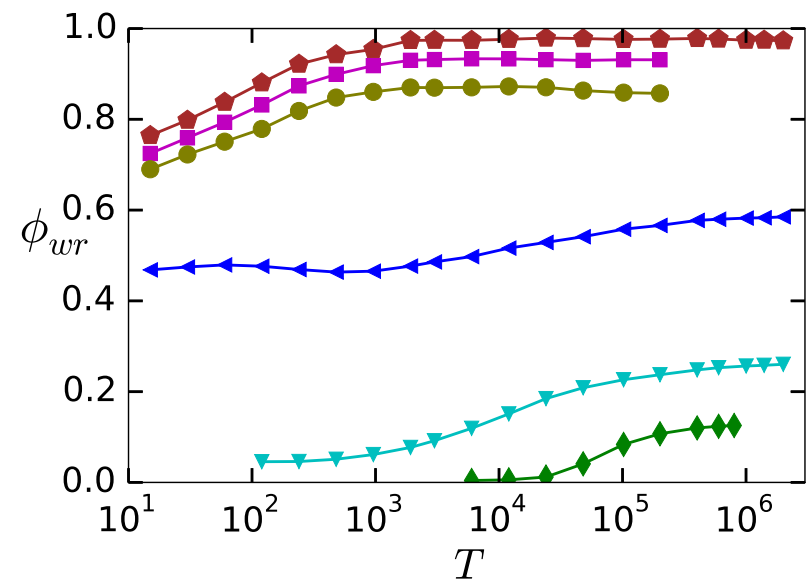

(a)

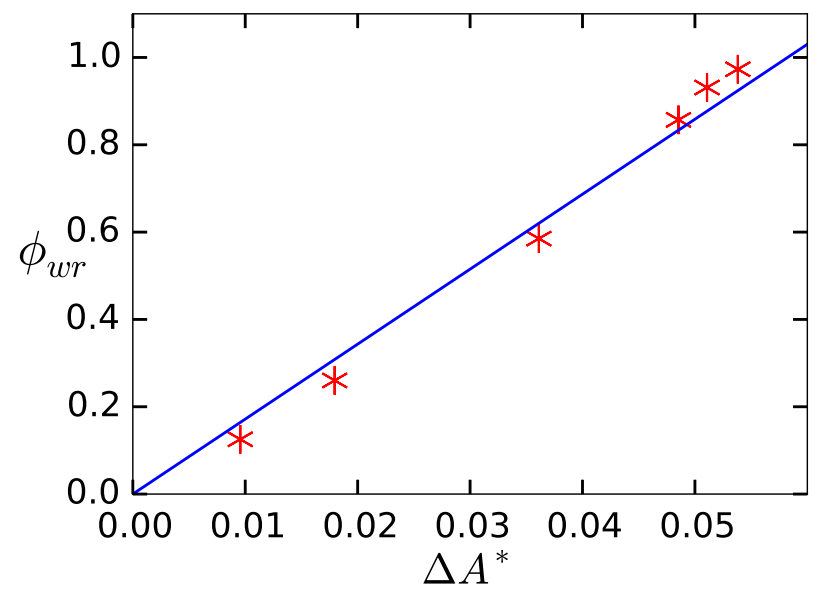

(b)

Fig. 8 (Color online) Time-evolution of (a) the area fraction covered by wrinkle domains $\phi_{w r}$ for $L=400$ (top three curves) and $L=800$ (bottom three

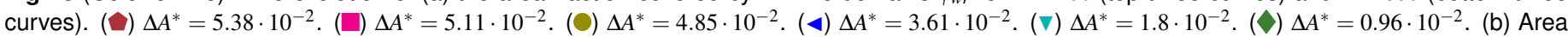
fraction covered by wrinkle domains at long simulation times. $(*)$ asymptotic value extracted from simulations. Blue line: Theoretical prediction from Eq. (29).

$e_{1 \text { roll }}^{n l}=0.4575 \pm 0.0025$. These numerical results in $1 \mathrm{D}$ confirm that $\Xi_{w r}$ in the roll phase vanishes at $\Sigma=\Sigma_{n l}$. Indeed, $\xi_{1 \text { roll }}^{n l}=e_{1 \text { roll }}^{n l}+$ $\Sigma_{n l} \alpha_{1 \text { roll }}^{n l} \approx(5.1 \pm 0.2) \cdot 10^{-3}$. In addition, we find $\lambda_{1 \text { roll }}^{n l} / \alpha_{1 \text { roll }}^{n l}=$ $17.17 \pm 0.23$. Using this result in the prediction Eq. (29) provides good agreement with simulations, as seen in Fig. 8(b).

Assuming that the transition to the frozen wrinkle state at large excess area corresponds to the filling of the system with the wrinkle state $\phi_{w r}=1$, we obtain a prediction for the critical excess area corresponding to the transition to regime C:

$$
\Delta A_{n l}^{*}=\frac{\alpha_{1 \text { roll }}^{n l}}{\lambda_{1 \text { roll }}^{n l}} .
$$

Using the numerical values reported above, we find $\Delta A_{n l}^{*}=$ $\alpha_{1 \text { roll }}^{n l} / \lambda_{1 \text { roll }}^{n l}=(5.83 \pm 0.78) \cdot 10^{-2}$ which is very close to the value of $\Delta A_{n l}^{*}$ observed in the simulations, Eq. (28).

\section{Large excess area}

Regime $\mathrm{C}$ is obtained for $\Delta A^{*}>\Delta A_{n l}^{*}$. In this regime, after some transient dynamics, the adhesion pattern is frozen into labyrinths as shown in Fig. 26. Such labyrinthine patterns have also been observed in the case of the $\mathrm{SH}$ equation 46 .

In our simulations of regime $\mathrm{C}$, the tension again converges to a stationary value. However, as opposed to the previous cases in regimes $\mathrm{A}$ and $\mathrm{B}$, the asymptotic tension depends on $\Delta A^{*}$. Furthermore, we observe in the simulations that, as the excess area $\Delta A^{*}$ increases in regime $\mathrm{C}$, the width of the wrinkles $\lambda=\lambda_{1 \text { roll }}$ decreases, whereas the maximum height and the variance of the membrane profile $\left\langle H^{2}\right\rangle$ increase.

In order to analyze this behavior, we perform the changes of variables $\chi=\zeta / \lambda_{1 \text { roll }}$, and $\eta=H /\left\langle H^{2}\right\rangle^{1 / 2}$. The excess area is then written as

$$
\Delta A^{*}=\frac{\left\langle H^{2}\right\rangle}{2 \lambda_{1 \text { roll }}^{2}} \int_{0}^{1} d \chi\left(\partial_{\chi} \eta\right)^{2}
$$

where the integral in the right hand side only depends on the shape of the wrinkle profile. An inspection of Eq. (31) shows that, if the shape of the wrinkle does not vary much, the quantity $\Delta A^{* 1 / 2} \lambda_{1 \text { roll }} /\left\langle H^{2}\right\rangle^{1 / 2}$ should be a constant. Indeed, the relation $\Delta A^{* 1 / 2} \lambda_{1 \text { roll }} /\left\langle H^{2}\right\rangle^{1 / 2} \approx 3.6$ is in good agreement with numerical results. Using the value of $\left\langle H^{2}\right\rangle^{1 / 2}$ measured in simulations, this expression provides the dashed magenta line on the main plot of Fig. 6 .

Assuming that the profile of the wrinkles is sinusoidal $H \sim$ $\sin \left(2 \pi \zeta / \lambda_{1 \text { roll }}\right)$, we find $\eta=2^{1 / 2} \sin (2 \pi \chi)$, and $\int_{0}^{1} d \chi\left(\partial_{\chi} \eta\right)^{2}=4 \pi^{2}$, suggesting that

$$
\frac{\Delta A^{* 1 / 2} \lambda_{1 \text { roll }}}{\left\langle H^{2}\right\rangle^{1 / 2}}=2^{1 / 2} \pi \approx 4.4 .
$$

The sine ansatz therefore leads to an overestimation of the constant, showing that the profile of the rolls is different from a simple sinusoidal profile.

Furthermore, the asymptotic value of the tension is controlled by the balance between the negative tension $\Sigma$ which tends to store excess area by forming the wrinkles, and the bending rigidity which tends to flatten the membrane. A simple balance between the two terms in the energy Eq. (18) suggests that $\Sigma H^{2} / \lambda_{1 \text { roll }}^{2} \sim$ $H^{2} / \lambda_{1 \text { roll }}^{4}$, or $\Sigma \sim 1 / \lambda_{1 \text { roll }}^{2}$. Simulation results reported in the inset of Fig. 6 indicate that this relation is in fair agreement with the numerical results with $\Sigma \lambda_{1 \text { roll }}^{2} \approx-88$.

If we assume again a sine profile for the membrane rolls, the potential energy density is not affected by the wavelength. The contributions that depend on the wavelength $\lambda_{1 \text { roll }}$ are the bending energy and the tension contribution. Minimizing the energy with respect to the wavelength, we find

$$
\Sigma \lambda_{1 \text { roll }}^{2}=-8 \pi^{2} \approx-79 .
$$

This relation between the tension and the wavelength appears in 
better agreement with the simulations as compared to Eq. (32), but is still not very accurate.

In the following, we proceed further with the sine ansatz to predict the wavelength, amplitude and tension from a direct minimization of the energy density. The details of the derivations are reported in Appendix E. Depending on the excess area, we find two regimes. In both regimes Eqs. (32)33 are valid. First, when the amplitude of the sine profile is small enough, the amplitude and the wavelength are both varied to minimise the energy density. However, for large enough excess area, the extrema of the sine profiles touch the walls. In this case, the short range repulsion potential $U_{d}$ prevents the membrane from crossing the wall. We therefore fix the amplitude and minimise the wavelength only. The crossover to this wall-contact regime occurs for $\Delta A^{*}=\Delta A_{w c}^{*}$ with

$$
\Delta A_{w c}^{*}=\frac{1}{8}\left(3-4 H_{m}^{2}\right)^{1 / 2}
$$

The details of the calculations are reported in Appendix E

The results of the sine-profile ansatz, shown on Fig. 9, reproduce the trends obtained with the full simulations in regime $\mathrm{C}$.

Note that the above value of $\Delta A_{w c}^{*}$ corresponds to a crossover rather than a sharp transition in the simulations. In addition, our convenient decomposition of the potential into a smooth doublewell potential and a sharp short-range repulsion is valid only when the minimum of the potential is far enough from the walls. In contrast, when $H_{m}$ is close to 1 , the short range repulsion $U_{d}$ affects the membrane profile in the potential well. One consequence of this is the inconsistency of Eq. (34) when $H_{m}>3^{1 / 2} / 2 \approx 0.866$. Our choice $H_{m}=0.7$ in simulations however corresponds to the regime where Eq. (34) is valid.

\section{Impermeable walls}

In this section, we discuss the limit of very impermeable walls with $\bar{v} \ll 1$. The full lubrication equations are reported in Appendix A These equations include not only a term accounting for the conservation of the total flow, as already found in a one-dimensional model ${ }^{24}$, but also a space-dependent tension that drives tangential forces along the membrane due to local area conservation. For the sake of simplicity, we neglect these two terms, and discuss a simplified model which is a straightforward transposition of Eq. (8,9) to the case of conserved dynamics:

$$
\begin{array}{r}
\partial_{t} h=\nabla\left(\mathscr{M}(h) \nabla\left(\kappa \Delta^{2} h-\sigma_{0} \Delta h+\mathscr{U}^{\prime}(h)\right)\right), \\
\sigma_{0}=\frac{\iint d x d y \nabla\left(\mathscr{M}(h) \nabla\left(\kappa \Delta^{2} h+\mathscr{U}^{\prime}(h)\right)\right) \Delta h}{\iint d x d y \nabla(\mathscr{M}(h) \nabla \Delta h) \Delta h} .
\end{array}
$$

The nonlinear mobility 24

$$
\mathscr{M}(h)=\frac{h_{0}^{3}}{24 \mu}\left[1-\frac{h^{2}}{h_{0}^{2}}\right]^{3}
$$

expresses the slowing down of the dynamics when the membrane approaches the wall at $h= \pm h_{0}$. This slowing down is caused by the increase of viscous dissipation when squeezing a thin liquid
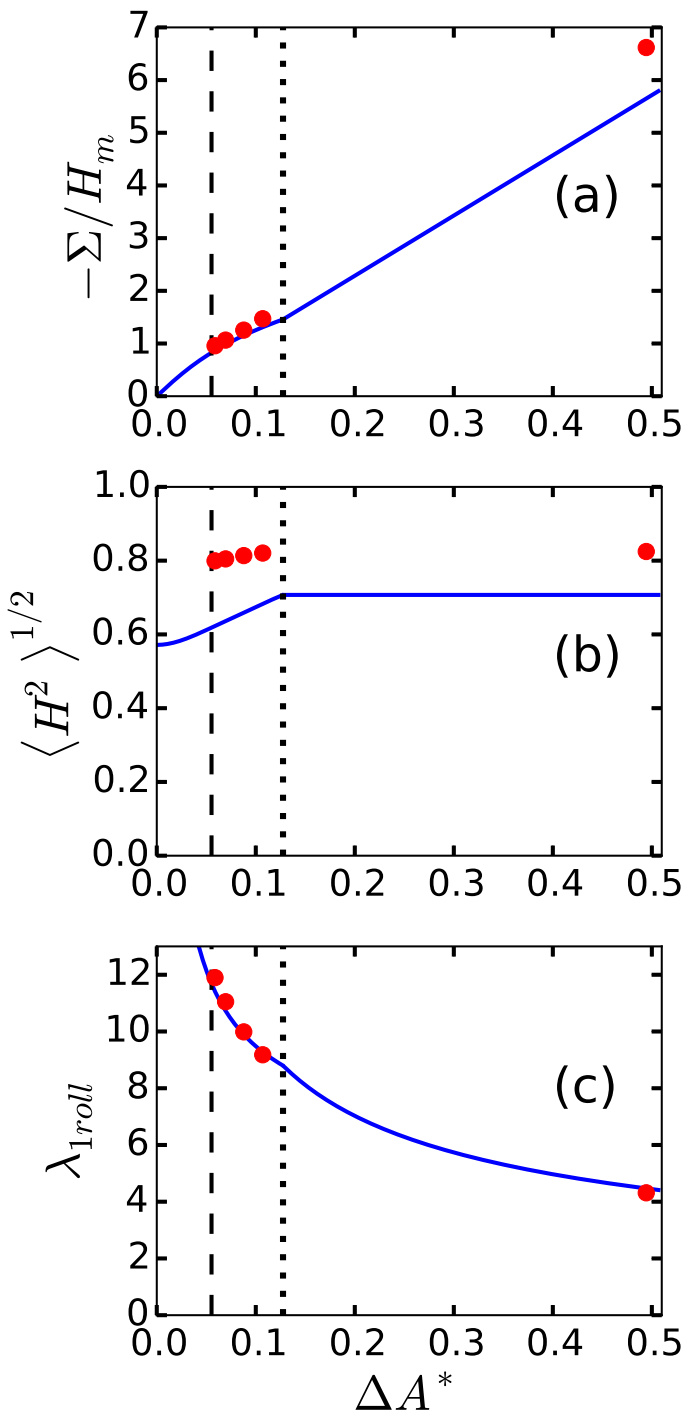

Fig. 9 (Color online) (a) Tension, (b) amplitude and (c) wavelength of the wrinkles in regime $\mathrm{C}$. Red dots represent the simulation results. The solid blue lines are the result of the sine-profile ansatz. The dashed and dotted vertical lines respectively indicate the start of regime $\mathrm{C}$ at $\Delta A^{*}=\Delta A_{n l}^{*}$, and the crossover to the wall-contact regime at $\Delta A^{*}=\Delta A_{w c}^{*}$. 


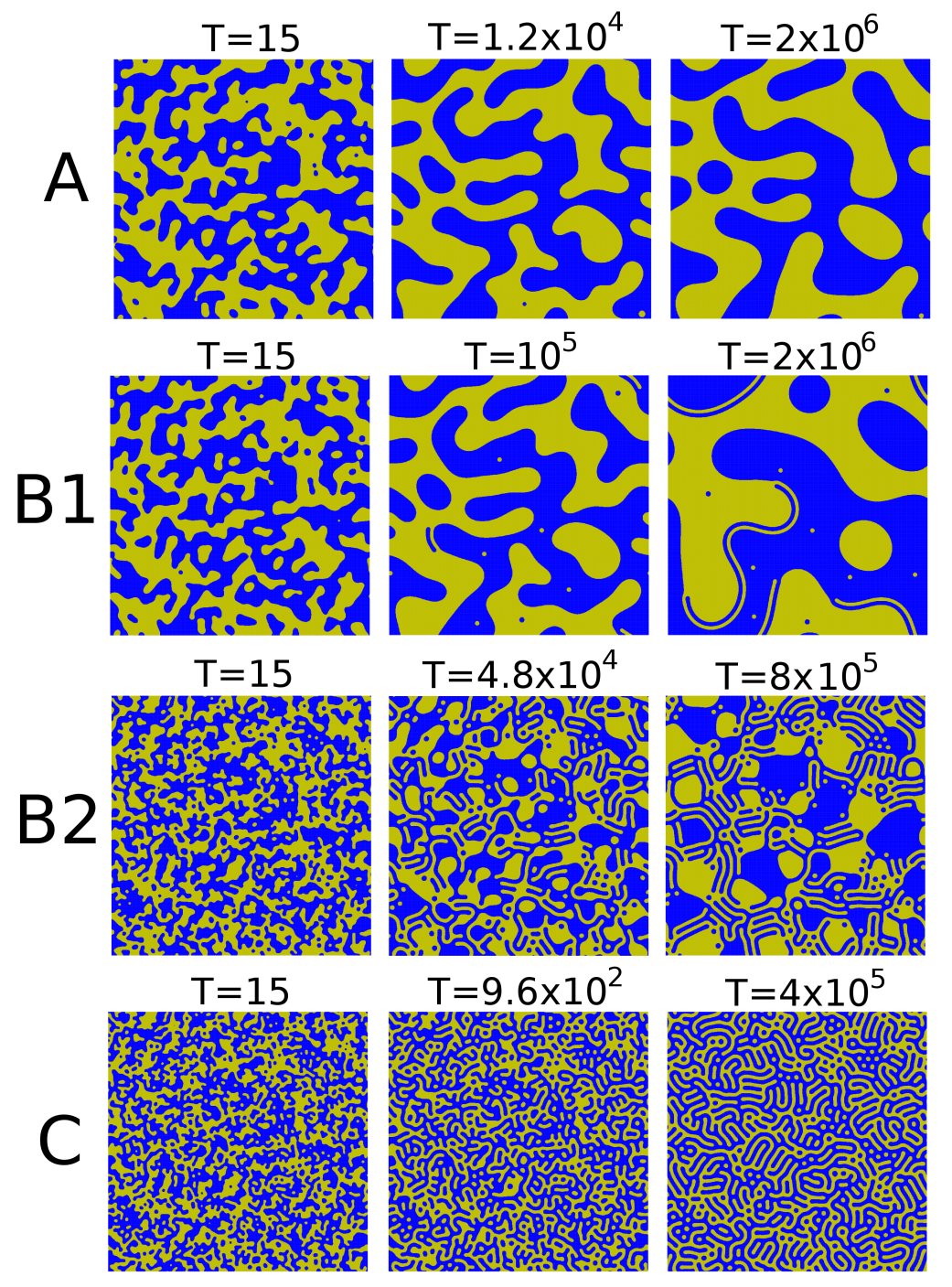

Fig. 10 (Color online) Membrane adhesion dynamics for impermeable wall for various excess areas. Yellow: adhesion patches on the upper wall. Dark blue: adhesion patches on the lower wall. Regime $A$ with freezing of flat domains for small excess area : $\Delta A^{*}=0.74 \times 10^{-2}$. Regime $B$ with coexistence of the flat-domain phase and wrinkle phase with coarsening for intermediate excess area: $\mathrm{B} 1$ with $\Delta A^{*}=0.88 \times 10^{-2}$, and $\mathrm{B} 2$ with $\Delta A^{*}=3.61 \times 10^{-2}$. Regime $C$ with frozen wrinkles for larger excess area $\Delta A^{*}=5.68 \times 10^{-2}$. System size $L \times L=400 \times 400$. 
film separating the membrane from the wall.

The simulations are performed in rescaled units that are defined in the same way as for the permeable limit, except for the rescaling of time $T=\mathscr{U}_{0}^{3 / 2} t /\left(24 \mu \kappa^{1 / 2}\right)$. In addition, we defined a rescaled mobility $M(H)=\left(1-H^{2}\right)^{3}$ via the relation $\mathscr{M}(h)=$ $M(H)\left(h_{0}^{3} /(24 \mu)\right)$.

We have performed simulations both with $M(H)=\left(1-H^{2}\right)^{3}$, and in the simplified case $M(H)=1$. Simulations performed with $L=$ 400 and with $M(H)=1$ exhibit a behavior similar to that obtained with $M(H)=\left(1-H^{2}\right)^{3}$ but are computationally ten times faster. This observation is consistent with previous simulations with the 1D model 26 . Thus, in order to minimise finite size and finite time effects, we have performed a systematic study with $M(H)=1$ for a system size $L=800$.

The evolutions of $\lambda_{\text {flat }}, \lambda_{w r}$ and $\Sigma$ are shown in Figs. 4(b) and 4(d) respectively. Due to the slow dynamics the results are less conclusive than the high permeability case. However, the simulations exhibit similar trends as in the large permeability limit, and we recover the three regimes $\mathrm{A}, \mathrm{B}$ and $\mathrm{C}$ discussed in the previous sections. The main differences are that (i) the dynamics is much slower, and (ii) we obtain a smaller value for the transition to coarsening $\Delta A_{c}^{*}=(0.4 \pm 0.03) \times 10^{-2}$ as compared to the large wall permeability limit.

Furthermore, as in the case of permeable walls, we find that tensionless dynamics, obtained by solving Eq. (35b) with $\Sigma=0$ leads to the same coarsening process as the Cahn-Hilliard equation, with the domain size growing as $t^{1 / 3}$. The results are reported in Fig 3

As a summary, the conserved dynamics discussed in this section using a simplification of the non-conserved case behaves in a way which is similar to the non-conserved case.

\section{Discussion}

\subsection{Finite size and initial conditions effects}

Since we do not have access to infinitely long times and infinitely large system sizes in simulations, we cannot make a final statement regarding the fact that frozen states do evolve slowly or are absolutely frozen at very long times. This is particularly important for the evaluation of $\Delta A_{c}$. Indeed, our observations show that the transition from regime A (frozen) to regime $B$ (coarsening) can be started via the formation of a single wrinkle resulting from the collision between two domain walls somewhere in the system. Thus, if the probability for such an event to occur per unit area is small but finite, then the threshold $\Delta A_{c}^{*}$ should decrease to zero as the system size increases to infinity.

To check the possible finite-size effects on $\Delta A_{c}^{*}$, we ran simulations for different system sizes $L=400$ and $L=800$ for small $\Delta A^{*}$. In both cases, we obtained the same values Eq. 27) for $\Delta A_{c}^{*}$ in large permeability limit. However, the case of impermeable walls seem to suffer stronger finite size effects. We indeed find a smaller value for the largest system $\Delta A_{c}^{*}=(0.4 \pm 0.03) \times 10^{-2}$ for $L=800$, as compared to $\Delta A_{c}^{*}=(0.81 \pm 0.07) \times 10^{-2}$ for $L=400$. Hence, our simulation sizes do not permit to reach a definitive conclusion about the existence of a finite limit for $\Delta A_{c}^{*}$ for infinitely large systems.

In addition, the probability to form wrinkles and to trigger the transition from the frozen regime A to regime B could be influenced by initial conditions. We have checked the sensitivity of the $A-B$ transition for $L=400$, using different random initial conditions described in Appendix C.2. We find a threshold which is similar, but slightly different $\Delta A_{c}^{*}=(0.835 \pm 0.035) \times 10^{-2}$ for the nonconserved model Eqs. 11,12 and $\Delta A_{c}^{*}=(0.77 \pm 0.03) \times 10^{-2}$ for the conserved model with constant mobility Eqs. 35a 35b with $M(H)=1$.

In summary, our simulations do not allow us to reach a conclusion with respect to the existence of regime A in an infinitely large system. It is however tempting to speculate that, based on the observation that the wrinkle phase can form as soon as a single wrinkle appears, regime A should lead to regime B for infinitely large systems for arbitrary low values of $\Delta A^{*}$. Note that any physical experiment will also be controlled by finite size effects and finite time observations. As a consequence, our simulations suggest that the frozen phase at low excess area (regime A) should be observable at least in finite size systems.

In contrast, our interpretation of the transition from regime $\mathrm{B}$ to regime $\mathrm{C}$ at high excess area is based on the filling of the system by the wrinkle phase. Such a transition is not triggered by an isolated event as in the transition to coarsening at low excess areas, and better self-averaging is expected, leading to smaller finite size effects, and little sensitivity to intial conditions.

To investigate finite-size effect on $\Delta A_{n l}^{*}$, we ran simulations for systems of different sizes $L=200$ and $L=400$ for large $\Delta A^{*}$. We obtain the same values $\Delta A_{n l}^{*}$ (given in Eq. (28) ) for both permeability limits. These results rule out the possibility of an influence of finite-size effect on the transition from regime $\mathrm{B}$ to regime $\mathrm{C}$.

\subsection{Labyrinthine pattern vs parallel rolls in the wrinkle phase}

In simulations, the wrinkle phase, which appears in regimes B and $C$ seems to be more disordered as the excess area is increased. Indeed, for very small excess areas in regime $B$, when all wrinkles are formed from a single initially isolated wrinkle, the wrinkle phase looks similar to a roll phase with a low density of defects. However, when the excess area increases, more defects appear in the wrinkle phase. Finally, in regime $C$ the wrinkle phase is composed of a disordered labyrinthine pattern.

In simulations of the $\mathrm{SH}$ equation by Le Berre et al.46, labyrinthine patterns were found for $\Sigma_{0}<\Sigma<\Sigma_{c}$ where $\Sigma_{0}=$ $-2.8284 H_{m}$. For $\Sigma<\Sigma_{0}$, these authors only found parallel-rolls. Moreover, Le Berre et al. 46 show that the roll pattern is always more stable than the labyrinthine pattern, i.e. rolls have lower energy. Hence, the labyrinthine pattern can be seen as a metastable state in which the system can be trapped.

In regime $\mathrm{B}$, we have $\Sigma>\Sigma_{0}$ at all times, indicating that the system can always be trapped in a meta-stable disordered state. Our results suggest that, although rolls are more stable, the order in the wrinkle phase is controlled by the domain-size on which the wrinkle phase is forming in regime B. Since this domain size decreases with increasing $\Delta A^{*}$, the related correlation length decreases, and disorder increases for increasing $\Delta A^{*}$ in the wrinkle phase of regime $B$. 
In regime $\mathrm{C}$, short-range disorder arises from the initial formation of microscopic domains, leading to a labyrinthine pattern. Since $\Sigma$ in steady-state decreases with increasing excess area $\Delta A^{*}$ in regime $\mathrm{C}$, we tried to increase $\Delta A^{*}$ to reach the roll phase. We therefore simulated a membrane with large excess area $\Delta A^{*}=0.4943$ in the limit of large permeability. In this case, the steady-state tension is found to be $\Sigma=-4.63<\Sigma_{0}$. However, the system still formed labyrinthine patterns and no parallel-rolls in our system. Note that the short-range repulsion $U_{d}$ could play an important role when the membrane area is large. Indeed, the membrane is then in contact with the wall as discussed in Sec. 8 and the contribution of $U_{d}$ should lead to deviations from the analogy to the simple SH equation.

\subsection{Conservation of the number of adhesion domains}

For all three regimes A, B and C, the number of domains decreases initially but is constant at long time. The time to reach the constant number of domains decreases as $\Delta A^{*}$ increases, as seen from Fig. 11(a).

The origin of this conservation can be traced back to the fact that the disappearance of domains by shrinking is stopped at small scales due to the formation of very stable localised structures. These localised structures are so stable that they are sometimes dragged on large distances by domain walls or wrinkles without loosing their integrity. Such stable localised structures have also been observed in the solution of the $\mathrm{SH}$ equation for parameters corresponding to the range of negative tensions relevant to our system 45 .

A detailed analysis of the localised structures is beyond the scope of our study. However, since the global evolution influences local dynamics via the tension $\Sigma$, it is tempting to propose a conditions under which the conservation of the number of domains should mainly be observed: (i) the domains are already well formed, i.e., there is no roughness or perturbation smaller than the domain wall width, and (ii) the tension is below some critical tension. From the plot of the number of positive and negative domains as a function of tension in Fig. 11(b), we find that the criterion $\Sigma<-0.552 \approx-0.79 H_{m}$ provides a condition under which the number of domains is always conserved in our simulations. The slow dynamics emerging after a short initial relaxation of the system, and which occurs with tensions around $\Sigma_{c}$ and $\Sigma_{n l}$ that are lower than this critical tension, corresponding to a regime where the number of domains is preserved.

\section{Conclusion}

In conclusion, we have developed a lubrication approach to study the dynamics of inextensible membranes confined between two flat attractive walls. We find that dynamics exhibit three types of regimes depending on the membrane excess area. For low excess area (regime A), the membrane freezes in a configuration with large adhesion patches on both walls. For intermediate area (regime B), the membrane exhibits coarsening, with a coexistence of flat adhesion domains with a wrinkle phase. For larger excess area, the membrane freezes into a labyrinthine wrinkle phase.

We hope that our results can provide hints for the understand- ing of the influence of confinement on the dynamics of model lipid membranes. On a more theoretical level, the model presented here defines a novel universality class for phase separation in two dimensions.

In order to gain further insight on the dynamics of confined membranes, the role of thermal fluctuations should be investigated. In one-dimensional models, such fluctuations were able to restore the coarsening by allowing the system to pass over the energy barriers which were trapping the system into metastable frozen states 26 . In addition, a study of the dynamics of the conserved model beyond the simplifications presented above is in order.

Finally, additional ingredients inspired from biological adhesion, such as the mobility and clustering of ligands and receptors ${ }^{14 / 48}$, or the active remodeling of the cytoskeleton 49.50 , could exhibit non-trivial coupling to the dynamics of the membrane presented here.

\section{Acknowledgments}

We acknowledge support from Biolub Grant No. ANR-12-BS040008. We thank Dr A. K. Tripathi for helpful discussions.

\section{A Lubrication limit for a membrane with area conservation}

In this section we outline the derivation of the equations which describe the dynamics of confined membranes in the lubrication limit. The derivation is analogous to the one-dimensional model by Le Goff et al. 24 . The two novel ingredients are: (i) the membrane is now two-dimensional, and (ii) we now enforce membrane area conservation.

\section{A.1 General derivation}

We apply the lubrication limit and the small-slope approximation to derive the dynamical equations of the membrane using the standard lubrication expansion. This expansion has been used in many studies of thin film dynamics, and generic details of the calculations can be found in Ref. [51. We assume a separation of scales

$$
x \sim \mathscr{O}\left(\varepsilon^{-1}\right), \quad y \sim \mathscr{O}\left(\varepsilon^{-1}\right), \quad h \sim \mathscr{O}(1), \quad,
$$

where $\varepsilon$ is a small parameter $(\varepsilon \ll 1)$. As a consequence, slopes are small $\partial_{x} h \sim \partial_{y} h \sim \varepsilon$.

To leading order in the Navier-Stokes equations, the velocity of the fluid takes the form of a Poiseuille flow

$$
\begin{aligned}
& v_{x}=\frac{z^{2}}{2 \mu} \partial_{x} p+a_{x} z+b_{x}, \\
& v_{y}=\frac{z^{2}}{2 \mu} \partial_{y} p+a_{y} z+b_{y},
\end{aligned}
$$

where $a_{x}, b_{x}, a_{y}, b_{y}$ and $p$ do not depend on $z$, but depend on $x$ and $y$.

Following the same lines as in Ref. $\stackrel{24}{ }$, we obtain an equation for the evolution of the membrane profile

$$
\partial_{t} h=-\nabla_{x y}\left\{\mathscr{M}_{z}(h) \nabla_{x y} f_{z}+\mathscr{M}_{\mathbf{j}}(h) \mathbf{j}+\mathscr{M}_{x y}(h) \mathbf{f}_{x y}\right\}+\frac{v}{2} f_{z},
$$




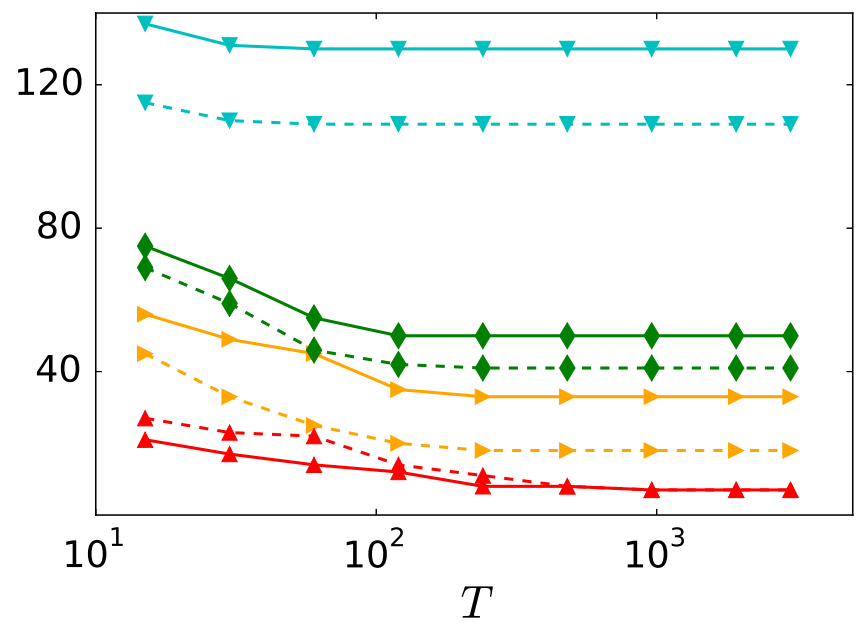

(a)

Fig. 11 (Color online) Number of positive domains (continuous line) and negative domains (dashed line) as a function of (a) time $T$ and (b) membrane

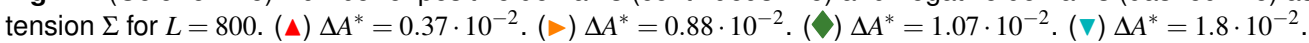

where $f_{z}$ and $\mathbf{f}_{x y}$ respectively denote the forces acting on the membrane along $z$ and in the $x y$ plane. In addition, we have defined the total flow

$$
\mathbf{j}=\int_{-h_{0}}^{h_{0}} d z \mathbf{v}_{x y}(z)
$$

Moreover, we have defined the functions

$$
\begin{aligned}
& \mathscr{M}_{z}(h)=\frac{h_{0}^{3}}{24 \mu}\left[1-\frac{h^{2}}{h_{0}^{2}}\right]^{3}, \\
& \mathscr{M}_{\mathbf{j}}(h)=\frac{1}{4} \frac{h}{h_{0}}\left[3-\frac{h^{2}}{h_{0}^{2}}\right], \\
& \mathscr{M}_{x y}(h)=-\frac{h_{0}^{2}}{8 \mu} \frac{h}{h_{0}}\left[1-\frac{h^{2}}{h_{0}^{2}}\right]^{2} .
\end{aligned}
$$

Then, using the fluid incompressibility and the boundary condition Eq. (6) we obtain

$$
0=\nabla_{x y} \cdot \mathbf{j}+2 v\left(\bar{p}-p_{\text {ext }}\right)
$$

where the average pressure $\bar{p}=\left(p_{+}+p_{-}\right) / 2$ obeys

$$
2 \nabla_{x y} \bar{p}=-\frac{3 \mu}{h_{0}^{3}} \mathbf{j}+\frac{1}{2} \frac{h}{h_{0}}\left[3-\frac{h^{2}}{h_{0}^{2}}\right] \nabla f_{z}+\frac{3}{2 h_{0}}\left[1-\frac{h^{2}}{h_{0}^{2}}\right] \mathbf{f}_{x y} .
$$

Taking the gradient of Eq. (42) and using (43), we obtain an equation for $\mathbf{j}$ without reference to pressure

$$
\nabla_{x y}\left(\nabla_{x y} \cdot \mathbf{j}\right)-\frac{3 \mu v}{h_{0}^{3}} \mathbf{j}=-\frac{v}{2} \frac{h}{h_{0}}\left[3-\frac{h^{2}}{h_{0}^{2}}\right] \nabla_{x y} f_{z}-\frac{3 v}{2 h_{0}}\left[1-\frac{h^{2}}{h_{0}^{2}}\right] \mathbf{f}_{x y} .
$$

We now need to evaluate the forces $f_{z}$ and $\mathbf{f}_{x y}$. In order to do so,

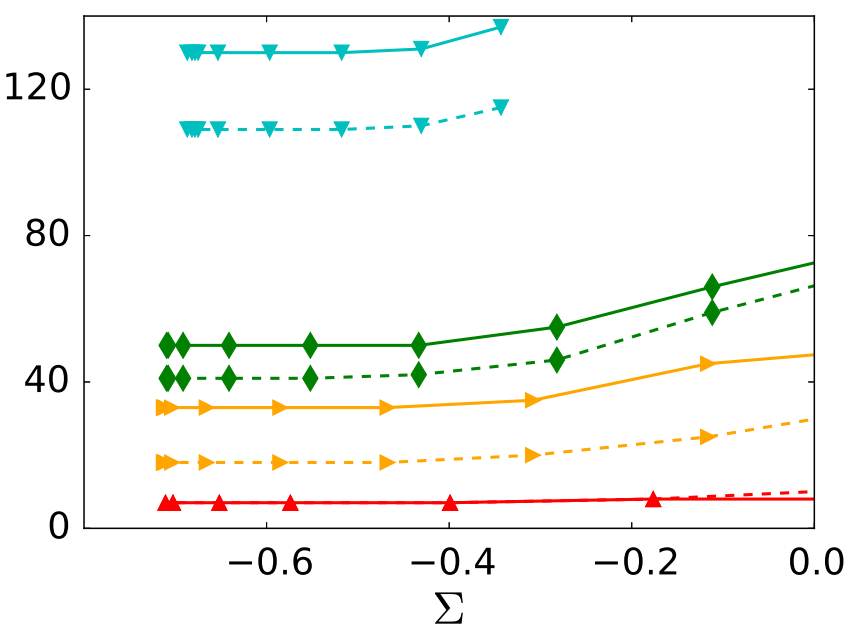

(b)

we write the variation of the energy $\mathscr{X}$

$$
\begin{aligned}
& \delta \mathscr{E}+\delta\left(\iint d \mathscr{A} \sigma\left(s_{1}, s_{2}\right)\right)=\iint d \mathscr{A}\left[\kappa\left(\Delta_{b} \mathscr{C}+\frac{\mathscr{C}^{3}}{2}-2 \mathscr{C} c_{G}\right) \mathbf{n}\right. \\
& \left.-\left(g^{i j} \partial_{s_{i}} \sigma \partial_{s_{j}} \mathbf{r}+\sigma \mathscr{C} \mathbf{n}\right)+\nabla \mathscr{U}(\mathbf{r})-g^{i j} \partial_{s_{i}} \mathscr{U}(\mathbf{r}) \partial_{s_{j}} \mathbf{r}-\mathscr{U}(\mathbf{r}) \mathscr{C} \mathbf{n}\right] \cdot \delta \mathbf{r},
\end{aligned}
$$

where $(i, j)$ can take values $(1,2),\left(s_{1}, s_{2}\right)$ are internal coordinates of membrane, $\Delta_{b}=g^{-1 / 2} \partial_{s_{j}}\left(g^{1 / 2} g^{i j} \partial_{s_{i}}\right)$ is the Beltrami laplacian, $g^{i j}$ is the inverse metric tensor, $\mathscr{C}$ is the mean curvature, $c_{G}$ is the Gaussian curvature and $\mathbf{n}$ is the unit vector normal to the membrane. At each point $\mathbf{r}\left(s_{1}, s_{2}\right)$ on the membrane surface we can define two tangent vectors

$$
\mathbf{t}_{i}=\partial_{s_{i}} \mathbf{r}\left(s_{1}, s_{2}\right),
$$

where $i=(1,2)$.

The resulting tangential and normal forces per unit surface are given by 52

$$
\begin{aligned}
f_{t_{j}}= & g^{i j} \partial_{s_{i}}(\sigma+\mathscr{U}(\mathbf{r}))-\nabla \mathscr{U}(\mathbf{r}) \cdot \mathbf{t}_{j}, \\
f_{n}=- & \kappa\left(\Delta_{b} \mathscr{C}+\frac{\mathscr{C}^{3}}{2}-2 \mathscr{C} c_{G}\right)+(\sigma+\mathscr{U}(\mathbf{r})) \mathscr{C} \\
& -\nabla \mathscr{U}(\mathbf{r}) \cdot \mathbf{n} .
\end{aligned}
$$

and in Eq. (4) of the main text, we use $\mathbf{f}=f_{n} \mathbf{n}+f_{t_{i}} \mathbf{t}_{i}$.

We are interested in physical conditions where the adhesion potential, the bending rigidity, and tension effects contribute simultaneously to the normal forces. Hence, we need to require that $\mathscr{U}_{0} / h_{0} \sim \kappa \varepsilon^{4} \sim \sigma \varepsilon^{2}$. In addition, since the normal force should balance the jump of pressure $\left(p_{+}-p_{-}\right) \sim \varepsilon^{-1}$ at the membrane from (4), we must require $f_{z} \sim f_{n} \sim \varepsilon^{-1}$. Combining these two conditions, we obtain that $\mathscr{U}_{0} \sim \varepsilon^{-1}, \kappa \sim \varepsilon^{-5}$, and $\sigma \sim \varepsilon^{-3}$. 
Using this scalings, and expanding $\sigma=\sigma_{0}+\sigma_{1}+\sigma_{2}+\ldots$, with $\sigma_{i} \sim \varepsilon^{-3+i}$, we find:

$$
\begin{aligned}
& f_{z}=-\kappa \Delta_{x y}^{2} h+\sigma_{0} \Delta_{x y} h-\mathscr{U}^{\prime}(h)+\mathscr{O}(1), \\
& \mathbf{f}_{x y}=\nabla_{x y} \sigma_{0}+\nabla_{x y} \sigma_{1}+\nabla_{x y} \sigma_{2}+\mathscr{O}(\varepsilon) .
\end{aligned}
$$

Here, we have kept the the sub-dominants contribution in the expression of the forces in the $x y$ plane for reasons that will become clear below.

We now use the membrane area conservation relation Eq. (2) which reads to leading order

$$
\nabla_{x y} \cdot \mathbf{v}_{x y}(h)=0
$$

where $\mathbf{v}_{x y}(h)$ is the $2 \mathrm{D}$ membrane velocity

$$
\mathbf{v}_{x y}=\mathscr{N}_{\mathbf{j}}(h) \mathbf{j}+\mathscr{N}_{z}(h) \nabla_{x y} f_{z}+\mathscr{N}_{x y}(h) \mathbf{f}_{x y}
$$

where

$$
\begin{aligned}
& \mathscr{N}_{\mathbf{j}}(h)=\frac{3}{4 h_{0}}\left[1-\frac{h^{2}}{h_{0}^{2}}\right], \\
& \mathscr{N}_{z}(h)=-\frac{h_{0}^{2}}{8 \mu} \frac{h}{h_{0}}\left[1-\frac{h^{2}}{h_{0}^{2}}\right]^{2}, \\
& \mathscr{N}_{x y}(h)=\frac{h_{0}}{8 \mu}\left[1+3 \frac{h^{2}}{h_{0}^{2}}\right]\left[1-\frac{h^{2}}{h_{0}^{2}}\right] .
\end{aligned}
$$

Inserting the expression of the forces Eqs. (48), (49) in Eq. (50), we see that the dominant contribution comes from the term $\sigma_{0}$ and reads

$$
\nabla_{x y} \cdot\left[\mathscr{N}_{x y}(h) \nabla_{x y} \sigma_{0}\right]=0 .
$$

Since periodic boundary conditions are used in this study, we conclude that $\sigma_{0}$ is necessarily a constant in space from the strong maximum principle 53 . Note however, that this is not constant in time. To sub-dominant order, the same equations are obtained and as a consequence $\sigma_{1}$ is also a constant in space.

To the next order, the membrane area conservation Eq. (2) still takes the form Eq. (50). Inserting the expression of the forces Eqs. (48), (49) into Eq. (50) then leads to

$$
0=\nabla_{x y} \cdot\left[\mathscr{N}_{\mathbf{j}}(h) \mathbf{j}+\mathscr{N}_{z}(h) \nabla_{x y} f_{z}+\mathscr{N}_{x y}(h) \nabla_{x y} \sigma_{2}\right] .
$$

Note also that Eq. (39) can be written as

$$
\begin{array}{r}
\partial_{t} h=-\nabla_{x y}\left\{\frac{h_{0}^{3}}{24 \mu}\left[1-\frac{h^{2}}{h_{0}^{2}}\right]^{3} \nabla_{x y} f_{z}+\frac{1}{4} \frac{h}{h_{0}}\left[3-\frac{h^{2}}{h_{0}^{2}}\right] \mathbf{j}\right. \\
\left.-\frac{h_{0}^{2}}{8 \mu} \frac{h}{h_{0}}\left[1-\frac{h^{2}}{h_{0}^{2}}\right]^{2} \nabla_{x y} \sigma_{2}\right\}+\frac{v}{2} f_{z} .
\end{array}
$$

Solving this latter equation requires the knowledge of $\sigma_{0}(t)$, $\mathbf{j}(x, y, t)$, and $\sigma_{2}(x, y, t)$ at each time.

The expression of $\sigma_{0}$ is found from the global conservation of the excess area Eq. (??). Indeed, from $\partial_{t} \Delta \mathscr{A}=0$, using periodic boundary conditions, we have

$$
0=\iint d x d y \partial_{t} h \Delta h
$$

which provides an expression for $\sigma_{0}$ as a function of $\mathbf{j}$ and $\sigma_{2}$.

Then, the two equations (54) and (44) provide a linear system of differential equations with space-dependent coefficients and no time-derivative, which must be solved at each time to obtain $\mathbf{j}$ and $\sigma_{2}$.

\section{A.2 Limit of very permeable walls}

In the limit of very permeable walls, the only term that survives in Eq. (55) is the last one $(v / 2) f_{z}$. Hence, the sub-dominant and space-dependent tension $\sigma_{2}(x, y, t)$ is irrelevant. The leading-order space-independent tension $\sigma_{0}(t)$ calculated from Eq. (56) leads to Eq. (9).

\section{A.3 Limit of impermeable walls}

In this case, the last term in Eq. (55) proportional to $v$ is negligible.

In addition, the total flow $\mathbf{j}$ obeys a simplified equation as compared to (44):

$$
\nabla_{x y} \cdot \mathbf{j}=0
$$

This equation is expressing that the total mass of liquid is then locally conserved. Taking the curl of Eq. (43), we obtain a second equation

$$
\begin{aligned}
& \frac{3 \mu}{h_{0}^{3}} \nabla_{x y} \times \mathbf{j} \\
& =\frac{3}{2}\left[1-\frac{h^{2}}{h_{0}^{2}}\right] \frac{\nabla h \times \nabla f_{z}}{h_{0}}-3 \frac{h}{h_{0}} \frac{\nabla h \times \nabla \sigma_{2}}{h_{0}^{2}} .
\end{aligned}
$$

These two scalar equations, together with Eq. (54) and suitable boundary conditions allow one to determine $\mathbf{j}$ and $\sigma_{2}$.

In the main text, we present simulations for a simplified conserved model, where $\sigma_{2}$ and $\mathbf{j}$ are neglected. Then, using Eq. (56) leads to the expression of $\sigma_{0}$ given in Eq. (35b).

\section{B Decrease of the total energy}

In this section we consider the general case of a membrane of height $h$ with the energy

$$
\mathscr{E}=\int d \mathscr{A} g
$$

where $g$ is an energy density depending on $h$ and its derivatives, and the related force

$$
f=\frac{\delta \mathscr{E}}{\delta h}
$$

The dynamics is ruled by one of the following equations

$$
\begin{aligned}
& \partial_{t} h=-f+\sigma_{0} \Delta h, \\
& \partial_{t} h=\nabla \cdot\left[\mathscr{M}(h) \nabla\left(f-\sigma_{0} \Delta h\right)\right],
\end{aligned}
$$


where $\mathscr{M}(h)$ is a positive nonlinear mobility depending on $h$, and the space-independent tension $\sigma_{0}$ enforces the total area conservation of the membrane (see Appendix A). In the current study, these two situations correspond respectively to the limits of very permeable walls and impermeable walls of Eq. (55).

As discussed in Appendix A area conservation is enforced by the relation

$$
\sigma_{0}=\frac{\int d \mathscr{A} f \Delta h}{\int d \mathscr{A}(\Delta h)^{2}}
$$

In the limit of very permeable walls described by Eq. (61), we have

$$
\begin{aligned}
\partial_{t} \mathscr{E} & =\int d \mathscr{A} f \partial_{t} h \\
& =-\int d \mathscr{A} f^{2}+\int d \mathscr{A} f \sigma_{0} \Delta h \\
& =-\int d \mathscr{A} f^{2}+\frac{\left(\int d \mathscr{A} f \Delta h\right)^{2}}{\int d \mathscr{A}(\Delta h)^{2}}
\end{aligned}
$$

Then, from the Schwarz inequality we have

$$
\left(\int d \mathscr{A} f \Delta h\right)^{2} \leq \int d \mathscr{A}(\Delta h)^{2} \int d \mathscr{A} f^{2}
$$

leading to $\partial_{t} \mathscr{E} \leq 0$.

In the opposite limit of impermeable walls described by Eq. (62), we now have area conservation is imposed via the relation

$$
\sigma_{0}=\frac{\int d \mathscr{A} \nabla \cdot[\mathscr{M}(h) \nabla f] \Delta h}{\int d \mathscr{A} \nabla \cdot[\mathscr{M}(h) \nabla(\Delta h)] \Delta h} .
$$

This expression is identical to that reported in the main text in Eq. (35b). Using integration by parts and periodic boundary conditions, this leads to

$$
\begin{aligned}
\partial_{t} \mathscr{E}= & -\int d \mathscr{A} \mathscr{M}(h)(\nabla f)^{2} \\
& +\frac{\left[\int d \mathscr{A} \mathscr{M}(h) \nabla f \cdot \nabla(\Delta h)\right]^{2}}{\int d \mathscr{A} \mathscr{M}(h)[\nabla(\Delta h)]^{2}} .
\end{aligned}
$$

Using once again the Schwarz inequality, we find $\partial_{t} \mathscr{E} \leq 0$.

\section{Numerical methods}

\section{C.1 Area conservation}

We choose a numerical method to determine $\Sigma$ which minimises the error on area conservation. In practice, we impose that $\Delta A_{T+d T}=\Delta A_{T}$. Since both in the conserved and non-conserved regimes the quantity $H_{T+d T}$ is linear in $\Sigma$, and since $\Delta A_{T+d T}$ is quadratic in $H_{T+d T}$, the conservation of the excess area implies the solution of a quadratic equation for $\Sigma$. This quadratic equation has two solutions. We choose the physically relevant solution, which is the one which is the closest to the value of $\Sigma$ calculated via a direct estimate Eq. (12) using $H=H_{T}$.

Our scheme can be seen as a specific discretization of Eq. (12) using a combination of $H_{T}$ and $H_{T+d T}$. The resulting variations in $\Delta A$ are $\sim 10^{-10}$.

\section{C.2 Initial conditions}

Our simulation scheme with area conservation requires a smooth initial condition for the excess area to be well defined. We have generated random smooth initial conditions with different excess area $\Delta A^{*}$ using two different methods. The first method uses the solution of the the time-dependent Ginzburg-Landau (TDGL) equation

$$
\partial_{T} H=w^{2} \Delta H-U^{\prime}(H),
$$

using an explicit scheme with finite-differences and random initial conditions. For each value of $w$, we take the membrane profile corresponding to the maximum value of $\Delta A^{*}$ as an initial condition for our simulation. To verify that this procedure does not affect the dynamics, we have repeated it solving the TDGL4 equation

$$
\partial_{T} H=-w^{4} \Delta^{2} H-U^{\prime}(H) .
$$

The final results were similar when considering similar initial $\Delta A^{*}$.

\section{C.3 Short-range repulsion near the walls}

Since the membrane does not approach the walls too much for $\Delta A^{*}<\Delta A_{n l}^{*}$, we have used the interaction potential without $U_{d}$.

For $\Delta A^{*} \geq \Delta A_{n l}^{*}=(5.53 \pm 0.15) \cdot 10^{-2}$, we use the double well potential with a short-range repulsion potential $U_{d}$ near the wall to prevent the membrane height from crossing the walls at $Z= \pm 1$, with $U_{0}=1$ and $d=0.01$. This requires a smaller $d T$ for numerical stability.

\section{C.4 Evaluation of the lengthscales in simulations}

We calculate the typical lengths of flat domains $\lambda_{\text {flat }}$ and wrinkle domains $\lambda_{w r}$ from the image of the membrane profile. First, we remove the localised structures. Next, we label positive regions $(H>0)$ by 2 and negative regions $(H<0)$ by 1 . Then, we erode the boundaries of domains 1 and 2 with a disk of radius $\theta=4$. This procedure removes domain walls. In addition, since this radius is larger than the half-width of the wrinkles, the procedure also subtracts the wrinkles regions from the zones with labels 1 or 2 . The eroded zones are labeled by 0 . The typical length of flat domains is given by

$$
\lambda_{\text {flat }}=\frac{A_{\text {flat }}}{L_{\text {flat }}},
$$

where $A_{\text {flat }}$ is the total area formed by domains 1 and 2. $L_{\text {flat }}$ is the total length of the boundaries of domains 1 and 2 and is calculated using the Cauchy-Crofton formula ${ }^{54}$ with two perpendicular and two diagonal sets of parallel lines forming a grid.

Next, we erode the boundaries of domains 0 with a disk of radius $R=8$. This procedure removes the domain walls between the flat domains 1 and 2 from domains 0 . We calculate the typical length of wrinkles domains $\lambda_{w r}$ from the remaining 0-regions by

$$
\lambda_{w r}=\frac{A_{w r}}{L_{w r}},
$$

where $A_{w r}$ is the total area formed by 0-domains. $L_{w r}$ is the total length of the boundaries of the domains 0 and is again calculated using the Cauchy-Crofton formula 54 . The expressions $(70,71$ are used in the main text in regime $\mathrm{B}$. 
In regime $\mathrm{A}$, we evaluate $\bar{\lambda}_{\text {flat }}$ defined from Eq. (24). In order to determine this quantity, we first notice that after eroding flat domains with the disc, the total length of the boundaries of flat domains $L_{D W}$ is doubled, thus $L_{\text {flat }} \approx 2 L_{D W}$. Moreover, during the disc erosion step the typical domain area is reduced by an amount $2 L_{D W} \theta$, where $\theta=4$ is the erosion disc radius. This leads to $A_{\text {syst }}=$ $A_{\text {flat }}+2 \theta L_{D W}$. Combining these relations, we obtain

$$
\bar{\lambda}_{\text {flat }}=\frac{A_{\text {syst }}}{L_{D W}} \approx 2\left(\lambda_{\text {flat }}+\theta\right)
$$

which is used in the main text.

\section{Motion by curvature in the large perme- ability limit}

Consider a domain wall between two opposite flat domains. To leading order for small domain wall curvature $K$, the Laplacian operator can be expanded as $\Delta \approx \partial_{\zeta \zeta}+K \partial_{\zeta}$, where $\zeta$ is a local coordinate along the normal to the domain wall. Expanding Eq. (11), we obtain

$$
-V_{n} \partial_{\zeta} H \approx-\partial_{\zeta}^{4} H-2 K \partial_{\zeta}^{3} H+\Sigma \partial_{\zeta}^{2} H+\Sigma K \partial_{\zeta} H-U^{\prime}(H),
$$

where $V_{n}$ is the normal front velocity. Multiplying both sides of Eq. (73) by $\partial_{\zeta} H$ and integrating with respect to $\zeta$ we find

$$
V_{n}=-\frac{1}{\alpha_{D W}}\left(\left[U_{0}\right]_{-}^{+}+K \xi_{D W}\right)
$$

where

$$
U_{0}=U(H)+\frac{1}{2} \partial_{\zeta \zeta}\left(\partial_{\zeta} H\right)^{2}-\frac{3}{2}\left(\partial_{\zeta \zeta} H\right)^{2}-\frac{\Sigma}{2}\left(\partial_{\zeta} H\right)^{2}
$$

accounts for interactions between domain walls, as discussed in Ref. 25 . Here, as in the main text $\alpha_{D W}=(1 / 2) \int d \zeta\left(\partial_{\zeta} H\right)^{2}$, and [ $]_{-}^{+}$ denotes the difference between the value of a given quantity in the adjacent adhesion domains on both sides of the domain wall. Moreover, $\xi_{D W}$ is the $\Xi$-energy of a flat domain wall per unit length

$$
\xi_{D W}=\int d \zeta\left\{\frac{1}{2}\left(\partial_{\zeta}^{2} H\right)^{2}+U(H)+\frac{\Sigma}{2}\left(\partial_{\zeta} H\right)^{2}\right\} .
$$

To leading order, area conservation imposes

$$
\begin{aligned}
0 & =\partial_{T}\left(L_{D W} \int d \zeta\left(\partial_{\zeta} H\right)^{2}\right) \\
& =-\int d \zeta\left(\left[U_{0}\right]_{-}^{+} K+\xi_{D W} K^{2}\right)+L_{D W} \partial_{T} \alpha_{D W}
\end{aligned}
$$

Using the chain rule $\partial_{T} \alpha_{D W}=\partial_{T} \Sigma \partial_{\Sigma} \alpha_{D W}$, we find an evolution equation for the tension Eq. 26.

\section{E Sine-profile ansatz in Regime C}

Here, we use a sine-profile ansatz

$$
H(\zeta)=a \cos (q \zeta)
$$

where $q=2 \pi / \lambda_{1 \text { roll }}$ to model the wrinkle phase. In normalised coordinates, the $\Xi$-energy per unit length of wrinkle then reads

$$
\xi_{1 \text { roll }}=\frac{\pi}{q}\left[\left(\frac{q^{4}}{2}+\frac{\Sigma q^{2}}{2}-\frac{H_{m}^{2}}{2}\right) a^{2}+\frac{3 a^{4}}{16}+\frac{H_{m}^{4}}{4}\right] .
$$

Minimizing the energy density $\xi_{1 \text { roll }} / \lambda_{1 \text { roll }}$ with respect to $a$ and $q$, we find

$$
\begin{aligned}
\Delta A^{*} & =\frac{-\Sigma H_{m}^{2}}{6}\left[1+\left(\frac{-\Sigma}{2 H_{m}}\right)^{2}\right], \\
\left\langle H^{2}\right\rangle & =\frac{2 H_{m}^{2}}{3}\left[1+\left(\frac{-\Sigma}{2 H_{m}}\right)^{2}\right], \\
\lambda_{1 \text { roll }}^{2} & =-8 \frac{\pi^{2}}{\Sigma} .
\end{aligned}
$$

The value of $\Sigma$ is obtained from the solution of the first equation. Then, $\left\langle H^{2}\right\rangle$ and $\Sigma$ are obtained from the two other equations. The above results correspond to the non-contact regime where $\Delta A^{*}<$ $\Delta A_{w c}^{*}$, and $a<1$. The value of the critical tension $\Delta A_{w c}^{*}$ is obtained from the condition $a=2^{1 / 2}\left\langle H^{2}\right\rangle^{1 / 2}=1$. The expression of $\Delta A_{w c}^{*}$ is provided in Eq. (34).

In wall-contact regime for $\Delta A^{*}>\Delta A_{w c}^{*}$, we set $a=1$, and minimise the energy density $\xi_{1 \text { roll }} / \lambda_{1 \text { roll }}$ with respect to $q$ only. This leads to

$$
\begin{aligned}
\left\langle H^{2}\right\rangle & =\frac{1}{2}, \\
\Sigma & =-8 \Delta A^{*}, \\
\lambda_{1 \text { roll }} & =\frac{\pi}{\Delta A^{* 1 / 2}} .
\end{aligned}
$$

\section{References}

1 D. Boal, Mechanics of the cell, Cambridge University Press, Cambridge, UK, 2002.

2 A. B. Hills, Internal Medicine Journal, 2002, 32, 170.

3 A. B. Hills, Internal Medicine Journal, 2002, 32, 242.

4 C. Das, M. G. Noro and P. D. Olmsted, Biophysical Journal, 2009, 97, $1941-1951$.

5 W. Helfrich, Zeitschrift für Naturforschung C, 1973, 28, 693703.

6 P. B. Canham, Journal of Theoretical Biology, 1970, 26, 61 81.

7 U. Seifert, Eur. Phys. J. B, 1999, 8, 405-415.

8 B. Kaoui, G. Biros and C. Misbah, Phys. Rev. Lett., 2009, 103, 188101.

9 J. O. Rädler, T. J. Feder, H. H. Strey and E. Sackmann, Phys. Rev. E, 1995, 51, 4526-4536.

10 O. Pierre-Louis, Phys. Rev. E, 2008, 78, 021603. 
11 F. Blachon, F. Harb, B. Munteanu, A. Piednoir, R. Fulcrand, T. Charitat, G. Fragneto, O. Pierre-Louis, B. Tinland and J.-P. Rieu, Langmuir, 2017, 33, 2444-2453.

12 R. Bruinsma, A. Behrisch and E. Sackmann, Phys. Rev. E, 2000, 61, 4253-4267.

13 K. Sengupta and L. Limozin, Phys. Rev. Lett., 2010, 104, 088101

14 E. Sackmann and A.-S. Smith, Soft Matter, 2014, 10, 1644 1659.

15 J. Brugués, B. Maugis, J. Casademunt, P. Nassoy, F. Amblard and P. Sens, Proceedings of the National Academy of Sciences, 2010, 107, 15415-15420.

16 G. T. Charras, M. Coughlin, T. J. Mitchison and L. Mahadevan, Biophysical Journal, 2008, 94, 1836 - 1853.

17 E. Paluch, M. Piel, J. Prost, M. Bornens and C. Sykes, Biophysical Journal, 2005, 89, 724 - 733.

18 T. Speck and R. L. C. Vink, Phys. Rev. E, 2012, 86, 031923.

19 M. Asfaw, B. Różycki, R. Lipowsky and T. R. Weikl, EPL (Europhysics Letters), 2006, 76, 703.

20 J. Langer, Annals of Physics, 1971, 65, 53 - 86.

21 E. D. Siggia, Phys. Rev. A, 1979, 20, 595-605.

22 P. C. Hohenberg and B. I. Halperin, Rev. Mod. Phys., 1977, 49, 435-479.

23 J. W. Cahn and J. E. Hilliard, The Journal of Chemical Physics, 1958, 28, 258-267.

24 T. Le Goff, P. Politi and O. Pierre-Louis, Phys. Rev. E, 2014, 90, 032114.

25 T. Le Goff, O. Pierre-Louis and P. Politi, Journal of Statistical Mechanics: Theory and Experiment, 2015, 2015, P08004.

26 T. Le Goff, P. Politi and O. Pierre-Louis, Phys. Rev. E, 2015, 92, 022918.

27 R. M. Hornreich, M. Luban and S. Shtrikman, Phys. Rev. Lett., 1975, 35, 1678-1681.

28 F. Campelo and A. Hernández-Machado, The European Physical Journal E, 2006, 20, 37-45.

29 B. Kaoui, G. H. Ristow, I. Cantat, C. Misbah and W. Zimmermann, Phys. Rev. E, 2008, 77, 021903.

30 M. T. J. and M.-P. Florian, ChemPhysChem, 2009, 10, 23052315.

31 A. Boţan, L. Joly, N. Fillot and C. Loison, Langmuir, 2015, 31, 12197-12202.

32 D. Huster, A. Jin, K. Arnold and K. Gawrisch, Biophysical Journal, 1997, 73, $855-864$.
33 R. Fettiplace and D. A. Haydon, Physiological Reviews, 1980, 60, 510-550.

34 J. C. Mathai, S. Tristram-Nagle, J. F. Nagle and M. L. Zeidel, The Journal of General Physiology, 2008, 131, 69-76.

35 S. Richardson, Journal of Fluid Mechanics, 1972, 56, 609 - 618.

36 D. Raucher, T. Stauffer, W. Chen, K. Shen, S. Guo, J. D. York, M. P. Sheetz and T. Meyer, Cell, 2000, 100, $221-228$.

37 J. Henriksen, A. Rowat, E. Brief, Y. Hsueh, J. Thewalt, M. Zuckermann and J. Ipsen, Biophysical Journal, 2006, 90, 1639 1649.

38 K. I. Pakkanen, L. Duelund, K. Qvortrup, J. S. Pedersen and J. H. Ipsen, Biochimica et Biophysica Acta (BBA) - Biomembranes, 2011, 1808, 1947 - 1956.

39 M. Miron-Mendoza, J. Seemann and F. Grinnell, Biomaterials, $2010,31,6425-6435$.

40 J. H. Snoeijer, Physics of Fluids, 2006, 18, 021701.

41 P. S. Swain and D. Andelman, Phys. Rev. E, 2001, 63, 051911.

42 S. Cox and P. Matthews, Journal of Computational Physics, 2002, 176, $430-455$.

43 K. R. Elder, J. Viñals and M. Grant, Phys. Rev. Lett., 1992, 68, 3024-3027.

44 M. C. Cross and D. I. Meiron, Phys. Rev. Lett., 1995, 75, 21522155.

45 K. Ouchi and H. Fujisaka, Phys. Rev. E, 1996, 54, 3895-3898.

46 M. Le Berre, E. Ressayre, A. Tallet, Y. Pomeau and L. Di Menza, Phys. Rev. E, 2002, 66, 026203.

47 A. Hagberg, A. Yochelis, H. Yizhaq, C. Elphick, L. Pismen and E. Meron, Physica D: Nonlinear Phenomena, 2006, 217, 186 192.

48 S. F. Fenz, A.-S. Smith, R. Merkel and K. Sengupta, Soft Matter, 2011, 7, 952-962.

49 T. D. Pollard and J. A. Cooper, Science, 2009, 326, 1208 - 1212.

50 P. Bun, S. Dmitrieff, J. M. Belmonte, F. J. Nédélec and P. Lénárt, eLife, 2018, 7, 1 - 27.

51 A. Oron, S. H. Davis and S. G. Bankoff, Rev. Mod. Phys., 1997, 69, 931-980.

52 O.-Y. Zhong-can and W. Helfrich, Phys. Rev. A, 1989, 39, 52805288.

53 M. H. Protter and H. F. Weinberger, Maximum Principles in Differential Equations, Prentice Hall, Englewood Cliffs, NJ, USA, 1967.

54 J. Stawiaski, E. Decencière and F. Bidault, Proceedings of the $8^{\text {th }}$ International Symposium on Mathematical Morphology, Rio de Janeiro, Brazil, 2007, pp. 349 - 360. 